\title{
Quantum critical behavior and trap-size scaling of trapped bosons in a one-dimensional optical lattice
}

\author{
Massimo Campostrini and Ettore Vicari \\ Dipartimento di Fisica dell'Università di Pisa and I.N.F.N., \\ Sezione di Pisa, Largo Bruno Pontecorvo 2, I-56127 Pisa, Italy
}

(Dated: March 17, 2010)

\begin{abstract}
We study the quantum (zero-temperature) critical behaviors of confined particle systems described by the one-dimensional (1D) Bose-Hubbard model in the presence of a confining potential, at the Mott insulator to superfluid transitions, and within the gapless superfluid phase. Specifically, we consider the hard-core limit of the model, which allows us to study the effects of the confining potential by exact and very accurate numerical results. We analyze the quantum critical behaviors in the large trap-size limit within the framework of the trap-size scaling (TSS) theory, which introduces a new trap exponent $\theta$ to describe the dependence on the trap size. This study is relevant for experiments of confined quasi $1 \mathrm{D}$ cold atom systems in optical lattices.

At the low-density Mott transition TSS can be shown analytically within the spinless fermion representation of the hard-core limit. The trap-size dependence turns out to be more subtle in the other critical regions, when the corresponding homogeneous system has a nonzero filling $f$, showing an infinite number of level crossings of the lowest states when increasing the trap size. At the $n=1$ Mott transition this gives rise to a modulated TSS: the TSS is still controlled by the trap-size exponent $\theta$, but it gets modulated by periodic functions of the trap size. Modulations of the asymptotic power-law behavior is also found in the gapless superfluid region, with additional multiscaling behaviors.

PACS numbers: 05.30.Rt, 05.30.Jp, 64.70.Tg, 67.85.-d
\end{abstract}

\section{INTRODUCTION}

The impressive progress in the experimental manipulation of cold atoms in optical lattices (see, e.g., Ref. [1] and references therein) have provided a great opportunity to investigate the interplay between quantum and statistical behaviors in particle systems. Cold atoms in optical lattices can be used to study many-body phenomena in dilute gases, such as quantum Mott-Hubbard transitions for bosonic atoms, see, e.g., Refs. [2-9]. An important feature of these experiments is the presence of a confining potential which traps the particles within a limited spatial region of the optical lattice created by laser-induced standing waves. The theoretical framework [10] is based on the Bose-Hubbard (BH) model [11] in the presence of a confining potential coupled to the particle density, i.e.,

$$
\begin{aligned}
H_{\mathrm{BH}} & =-\frac{J}{2} \sum_{\langle i j\rangle}\left(b_{i}^{\dagger} b_{j}+b_{j}^{\dagger} b_{i}\right)+\frac{U}{2} \sum_{i} n_{i}\left(n_{i}-1\right) \\
& +\mu \sum_{i} n_{i}+\sum_{i} V\left(r_{i}\right) n_{i},
\end{aligned}
$$

where $\langle i j\rangle$ is the set of nearest-neighbor sites, $n_{i} \equiv b_{i}^{\dagger} b_{i}$ is the particle density operator.

We consider a power-law trapping potential

$$
V(r)=v^{p} r^{p} \equiv(r / l)^{p},
$$

where $r \equiv|\vec{x}|, v$ and $p$ are positive constants and $l \equiv 1 / v$ is the trap size. Experiments are usually set up with a harmonic potential, i.e., $p=2$. Far from the origin the potential $V(r)$ diverges, therefore $\left\langle n_{i}\right\rangle$ vanishes and the particles are trapped. The inhomogeneity due to the trapping potential strongly affects the phenomenology of quantum transitions in homogeneous systems.

The homogeneous BH model without trap undergoes Mott insulator to superfluid quantum transitions driven by the chemical potential $\mu$, whose low-energy properties are described by a nonrelativistic $\mathrm{U}(1)$-symmetric bosonic field theory [11], which is characterized by the dynamic exponent $z=2 .{ }^{1}$ In the presence of a confining potential, theoretical and experimental results have shown the coexistence of Mott insulator and superfluid regions when varying the total occupancy of the lattice, see, e.g., Refs. [7, 10, 13-22]. However, at fixed trap size, the system does not develop a critical behavior with a diverging length scale $[13,17]$.

Criticality can be recovered only in the limit of large trap size. As put forward in Refs. [23, 24], this critical regime can be described in the framework of the trapsize scaling (TSS) theory, where the critical behavior is cast in the form a TSS with a nontrivial trap exponent $\theta$, which determines how the length scale of the critical modes at the critical point diverges with increasing trap size, i.e., $\xi \sim l^{\theta}$. For example, let us consider a standard scenario (see, e.g., Ref. [25]), in which the quantum $T=0$ transition of the the homogeneous $d$-dimensional system has one relevant parameter $\mu$, with critical value $\mu_{c}$. The

\footnotetext{
1 The special transitions at fixed integer density belong to a different universality class [11], described by a relativistic $\mathrm{U}(1)$ symmetric bosonic field theory, which is the $(d+1)$-dimensional $\mathrm{XY}$ universality class [12]. Thus its dynamic exponent is $z=1$.
} 
simplest TSS Ansatz [23] for the asymptotic behavior of the free-energy density in the presence of a confining potential (2) is

$$
F(\mu, T, l, x)=l^{-\theta(d+z)} \mathcal{F}\left(\bar{\mu} l^{\theta / \nu}, T l^{\theta z}, x l^{-\theta}\right),
$$

where $x$ is the distance from the middle of the trap, $\bar{\mu} \equiv$ $\mu-\mu_{c}, z$ is the dynamic exponent and $\nu \equiv 1 / y_{\mu}$ where $y_{\mu}$ is the renormalization-group (RG) dimension of $\mu$. Moreover, any low-energy scale at $T=0$, and specifically the gap, is expected to behave as

$$
\Delta=l^{-\theta z} \mathcal{D}\left(\bar{\mu} l^{\theta / \nu}\right) .
$$

The above TSS has been verified by analytical and accurate numerical calculations [23] within the quantum $\mathrm{XY}$ chain in a space-dependent transverse external field, which acts as a trap for the spinless fermions of its quadratic Hamiltonian representation, which can be obtained by a Jordan-Wigner transformation.

The general features of the TSS at the Mott to superfluid transitions in $d$-dimensional BH models have been discussed in Ref. [23]. Beside the dynamic critical exponent $z$ and the RG dimension $y_{\mu}$ of $\mu$, which control the critical behavior of the homogeneous system and can be determined from the corresponding continuum theory $[11,25]$, the TSS requires the trap exponent $\theta$, which can be derived by a RG analysis of the corresponding perturbation [23]. For one- and two-dimensional systems, the critical exponents entering the scaling formulas (3) and (4) are

$$
z=2, \quad y_{\mu} \equiv 1 / \nu=2, \quad \theta=p /(p+2),
$$

where $p$ is the power of the confining potential (2).

In this paper we investigate the quantum critical behaviors of the one-dimensional (1D) BH model at zero temperature in the presence of a confining potential, at the Mott to superfluid transitions, and within the gapless superfluid phase. The 1D BH model in the presence of a confining potential is of experimental relevance in optical lattices, where quasi $1 \mathrm{D}$ confined particle systems have been realized, see, e.g., Refs. [1, 4, 5, 9].

Specifically, we consider the hard-core limit, $U \rightarrow \infty$, of the 1D BH model, which allows us to study the effects of the confining potential by exact and very accurate numerical results. The hard-core limit implies that the particle number $n_{i}$ per site is restricted to the values $n_{i}=0,1$. In this limit the model can be mapped into the XX chain model with a space-dependent transverse external field,

$$
\begin{aligned}
H_{\mathrm{XX}} & =-J \sum_{i}\left(S_{i}^{x} S_{i+1}^{x}+S_{i}^{y} S_{i+1}^{y}\right) \\
& -\sum_{i}\left[\mu+V\left(x_{i}\right)\right] S_{i}^{z}
\end{aligned}
$$

where $S_{i}^{a}=\sigma_{i}^{a} / 2$ and $\sigma^{a}$ are the Pauli matrices, which are related to the boson operators $b_{i}$ by $\sigma_{i}^{x}=b_{i}^{\dagger}+b_{i}$, $\sigma_{i}^{y}=i\left(b_{i}^{\dagger}-b_{i}\right), \sigma_{i}^{z}=1-2 b_{i}^{\dagger} b_{i}$. In the following we fix $J=1$. Then, by a Jordan-Wigner transformation, one can further map it into a model of spinless fermions, see, e.g., Ref. [25].

In the absence of the trap, the 1D hard-core $\mathrm{BH}$ model has three phases: two Mott insulator phases, for $\mu>1$ with $\left\langle n_{i}\right\rangle=0$ and for $\mu<-1$ with $\left\langle n_{i}\right\rangle=1$, separated by a gapless superfluid phase for $|\mu|<1$. Therefore, there are two Mott insulator to superfluid transitions at $\mu=1$ and $\mu=-1$. At both transitions the exponents controlling the critical behavior are those reported in Eq. (5). The gapless superfluid phase is instead described by a free massless bosonic field theory with dynamic exponent $z=1$, see, e.g., Ref. [26].

The effects of the confining potential at the low-density transition $(\mu=1)$ has been already studied in Ref. [23], where TSS has been shown to emerge by analytical calculations exploiting the spinless fermion formulation of the 1D hard-core BH model.

In this paper we extend the study of the trap-size dependence to all critical regions of its phase diagram. We present results for the trap-size dependence in the gapless superfluid region and at the $n=1$ Mott insulator to superfluid transition.

For this purpose we exploit the free spinless fermion representation of the $1 \mathrm{D}$ hard-core $\mathrm{BH}$ model, which allows us to perform computations for very large systems, since they only require the diagonalization of a $L \times L$ matrix where $L$ is the number of lattice sites. We obtain numerical results for chains of size $L$, with a trap of size $l$ centered in the middle of the chain (unless explicitly stated, we consider odd $L$ s so that the middle of the trap coincides with the middle site of the chain); we choose $L$ large enough to have negligible finite- $L$ effects; we are able to obtain results correct to machine precision for $l$ up to $O\left(10^{3}\right)$. We then analyze the quantum critical behaviors in the presence of the trap within the framework of the TSS theory.

The trap-size dependence shows subtle effects in the parameter region where the homogeneous model without trap has a nonzero filling $f$, i.e., for $\mu<1$, therefore in the superfluid region and at the $n=1$ Mott transition. This is essentially related to the presence of level crossings at finite trap size. They arise because the particle number is conserved, i.e., the particle number operator $\hat{N}=\sum_{i} n_{i}$ commutes with the BH Hamiltonian (1) even in the presence of the trapping potential; thus the eigenvectors do not depend on $\mu$, even though the eigenvalues do. In the presence of the trapping potential (2), the particle number $N \equiv\langle\hat{N}\rangle$ is finite and increases as $N \sim l$ with increasing the trap size $l$. Therefore, as $l \rightarrow \infty$, there is an infinite number of ground-state level crossings where $N$ jumps by 1 and the gap $\Delta$ vanishes.

As we shall see, this phenomenon gives rise to a new interesting scenario at the $n=1$ Mott transition, requiring a revision of the TSS Ansatz (3) and (4) into a modulated TSS: the TSS is still controlled by the trap-size exponent $\theta=p /(p+2)$, as in the case of the low-density Mott 
transition, but it gets modulated by periodic functions of the trap size.

We provide numerical evidence of universality at the low-density and $n=1$ Mott transitions by considering a more general 1D hard-core BH model with nearestneighbor density-density interactions, corresponding to the so-called XXZ model. Since the corresponding fermion representation is not longer quadratic, the numerical results are obtained by using density matrix renormalization group (DMRG) methods.

We also show that the trap-size dependence in the gapless superfluid phase is characterized by power-law asymptotic behaviors which are modulated by periodic functions of the trap size. Moreover, it shows a multiscale behavior characterized by different length scales diverging with different power laws with increasing trap size, associated with the smooth modes and with the modes at the Fermi momentum $k_{F}=\pi f$.

The paper is organized as follows. In Sec. II we discuss the TSS at the low-density Mott transition in the 1D hard-core BH model. We extend the analysis of Ref. [23], providing the TSS of several observables and checking its universality within the XXZ model. In Sec. III we discuss the local density approximation to determine the particle density in the presence of the confining potential. In Sec. IV we study the trap-size dependence within the gapless superfluid phase, presenting results for the gap, the particle density and its correlators, the one-particle density matrix and the von Neumann entanglement entropy. In Sec. V we consider the $n=1$ Mott insulator to superfluid transition at $\mu=-1$. We present results at fixed trap size for the XX and XXZ models, and show that their trap-size dependence is described by a modulated TSS. Finally, in Sec. VI we draw our conclusions. In App. A we report some details on the numerical calculations presented in paper. App. B reports some results for the homogeneous $1 \mathrm{D}$ hard-core $\mathrm{BH}$ model with open boundary conditions, showing that modulated scaling behaviors of the gap, the particle density, and the subleading corrections of the entanglement entropy, are already present in the finite size behavior of the homogeneous model within the superfluid region; we provide exact formulae for the total particle number and for the particle density at the middle of the chain.

\section{TRAP-SIZE SCALING AT THE LOW-DENSITY MOTT TRANSITION}

\section{A. The TSS limit}

In order to show the existence of a nontrivial TSS limit around $\mu=1$, i.e., at the transition between a lowdensity superfluid and the empty vacuum state (which may be named $n=0$ Mott phase), we exploit the exact mapping of the XX model into a model of spinless fermions, by the Jordan-Wigner transformation

$$
\begin{aligned}
\sigma_{i}^{x} & =\prod_{j<i}\left(1-2 c_{j}^{\dagger} c_{j}\right)\left(c_{i}^{\dagger}+c_{i}\right), \\
\sigma_{i}^{y} & =i \prod_{j<i}\left(1-2 c_{j}^{\dagger} c_{j}\right)\left(c_{i}^{\dagger}-c_{i}\right), \\
\sigma_{i}^{z} & =1-2 c_{i}^{\dagger} c_{i},
\end{aligned}
$$

which leads to the Hamiltonian

$$
\begin{aligned}
& H_{c}=\sum_{i j} c_{i}^{\dagger} h_{i j} c_{j}, \\
& h_{i j}=\delta_{i j}-\frac{1}{2} \delta_{i, j-1}-\frac{1}{2} \delta_{i, j+1}+\left[\bar{\mu}+V\left(x_{i}\right)\right] \delta_{i j},
\end{aligned}
$$

with $\bar{\mu} \equiv \mu-1$. In the fermion representation the Hamiltonian can be easily diagonalized by introducing new canonical fermionic variables $\eta_{k}=\sum_{i} \phi_{k i} c_{i}$, where $\phi$ satisfies the equation

$$
h_{i j} \phi_{k j}=\omega_{k} \phi_{k i}
$$

obtaining

$$
H_{c}=\sum_{k} \omega_{k} \eta_{k}^{\dagger} \eta_{k}
$$

The ground state contains all $\eta$-fermions with $\omega_{k}<0$; the number of filled energy levels is $N$. The energy gap is

$$
\Delta=\min _{k}\left|\omega_{k}\right| .
$$

Since $\phi_{k i}$ is an orthogonal matrix, the expectation values of the $c$-operators can be obtained by using the inverse relation $c_{i}=\sum_{k} \phi_{k i} \eta_{k}$.

The above equations have a nontrivial TSS limit around

$$
\bar{\mu} \equiv \mu-1=0,
$$

i.e., at the transition between a low-density superfluid and the empty vacuum state. We consider the continuum limit of Eq. (9), by rewriting the discrete differences in terms of derivative expansions. Near the critical point $\bar{\mu}=0$ and for sufficiently small values of $k$ (this is required by the smoothness hypothesis underlying the continuum limit), we obtain

$$
\left[\bar{\mu}+(x / l)^{p}-\frac{1}{2} \frac{d^{2}}{d x^{2}}-\frac{1}{24} \frac{d^{4}}{d x^{4}}+\ldots\right] \phi_{k}(x)=\omega_{k} \phi_{k}(x),
$$

where $\phi_{k}(x) \equiv \phi_{k x}$. Then, by replacing

$$
\begin{aligned}
& x=l^{p /(2+p)} X, \\
& \bar{\mu}=l^{-2 p /(2+p)} \mu_{r}, \\
& \omega_{k}=l^{-2 p /(2+p)} \Omega_{k},
\end{aligned}
$$


and neglecting terms which are suppressed in the large- $l$ limit, we obtain

$$
\left(-\frac{1}{2} \frac{d^{2}}{d X^{2}}+X^{p}\right) \varphi_{k}(X)=\left(\Omega_{k}-\mu_{r}\right) \varphi_{k}(X) \equiv \bar{\Omega}_{k} \varphi_{k}(X),
$$

where $\varphi_{k}(X) \equiv \phi_{k}\left(l^{p /(2+p)} X\right)$; note that $\bar{\Omega}_{k}$ is independent of $\mu_{r}$. Recalling that $z=2$ and $y_{\mu}=2$ at this transition, we infer $\theta=p /(2+p)$, as also obtained by RG arguments [23]. Therefore, the solutions of Eq. (15) determine the TSS in the limit $\bar{\mu} \equiv \mu-1 \rightarrow 0$ and $l \rightarrow \infty$, keeping $\mu_{r} \equiv l^{2 \theta} \bar{\mu}$ and $X \equiv l^{-\theta} x$ fixed. On the other hand, subleading terms, such as the $d^{4} / d x^{4}$ term in the l.h.s. of Eq. (13), give rise to $O\left(l^{-2 \theta}\right)$ corrections in the TSS limit (14) of the Eq. (9). Thus, we expect that the TSS of any quantity is approached with $O\left(l^{-2 \theta}\right)$ scaling corrections.

For $p=2$, we obtain

$$
\begin{aligned}
& \bar{\Omega}_{k} \equiv \Omega_{k}-\mu_{r}=2^{1 / 2}(k+1 / 2), \quad k \geq 0, \\
& \varphi_{k}(X)=\frac{2^{1 / 8} H_{k}\left(2^{1 / 4} X\right)}{\pi^{1 / 4} 2^{k / 2}(k !)^{1 / 2}} \exp \left(-X^{2} / \sqrt{2}\right),
\end{aligned}
$$

where $X \equiv x / l^{1 / 2}$ and $H_{k}(x)$ are Hermite's polynomials $H_{k}(x)=(-1)^{k} e^{x^{2}} d^{k} e^{-x^{2}} / d x^{k}$.

For $p=4$, we can solve numerically Eq. (15) by Numerov's method; the resulting energy levels are $\bar{\Omega}_{0}=$ $0.667986, \bar{\Omega}_{1}=2.39364, \bar{\Omega}_{2}=4.69680, \bar{\Omega}_{3}=7.33573$, $\bar{\Omega}_{4}=10.2443, \bar{\Omega}_{5}=13.3793$, etc.; Bohr-Sommerfield quantization formula gives

$$
\begin{aligned}
\bar{\Omega}_{k} & \approx\left[\gamma_{4}(k+1 / 2)\right]^{4 / 3}, \\
\gamma_{4} & =\sqrt{\pi / 2} \Gamma(7 / 4) / \Gamma(5 / 4) \cong 1.27082,
\end{aligned}
$$

which is accurate to $0.1 \%$ already for $\bar{\Omega}_{5}$. For large $k$ and generic $p$, the semiclassical limit gives

$$
\bar{\Omega}_{k} \sim k^{2 p /(2+p)} .
$$

For $p \rightarrow \infty$, Eq. (15) becomes equivalent to the Schrödinger equation of a free particle in a box of size $L=2 l$ with boundary conditions $\varphi(-1)=\varphi(1)=0$, leading to

$$
\begin{aligned}
& \bar{\Omega}_{k}=\frac{\pi^{2}}{8}(k+1)^{2}, \quad k \geq 0, \\
& \varphi_{k}(X)=\sin \left[\frac{\pi}{2}(k+1)(X+1)\right],
\end{aligned}
$$

where $X \equiv x / l$.

\section{B. TSS of observables}

\section{The energy gap}

The existence of the TSS limit implies that any low energy scale behaves as $l^{-2 \theta} \mathcal{E}\left(\mu_{r}\right)$, where $\mathcal{E}\left(\mu_{r}\right)$ is a scaling

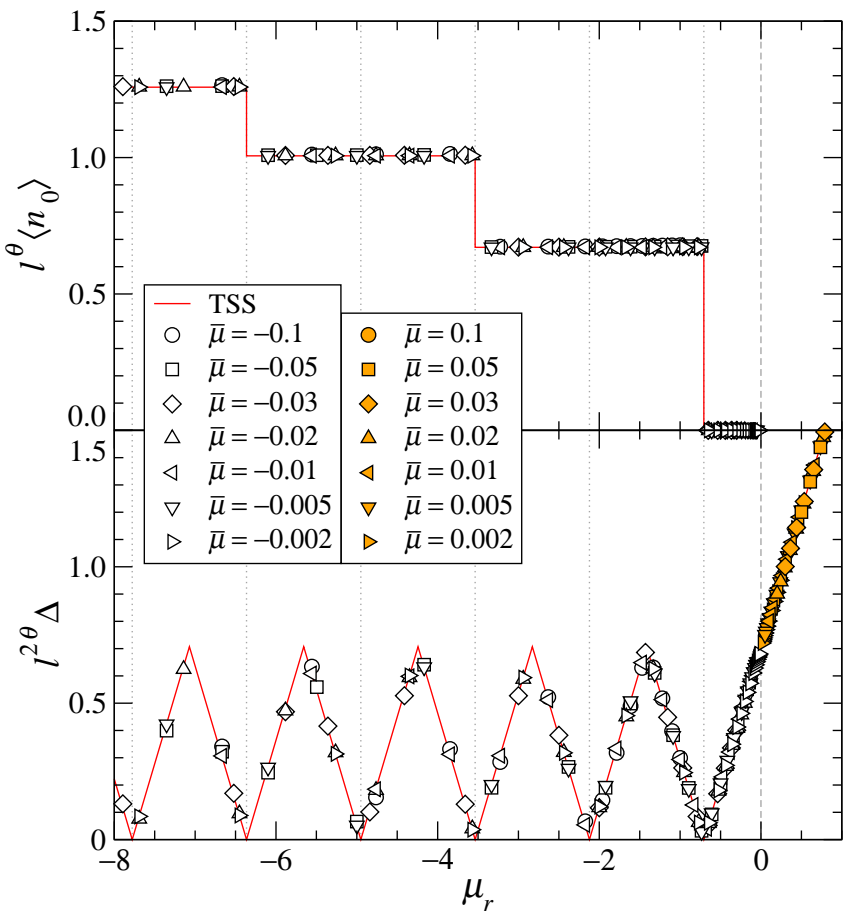

FIG. 1: The rescaled energy gap $l^{2 \theta} \Delta$ (below) and the rescaled particle density $l^{\theta}\left\langle n_{0}\right\rangle$ in the middle of the trap (above) for $p=2(\theta=1 / 2)$, compared with the predictions of TSS. We report results for several values of $\bar{\mu}$ and $l \gtrsim 10$. They are perfectly consistent with the analytical TSS.

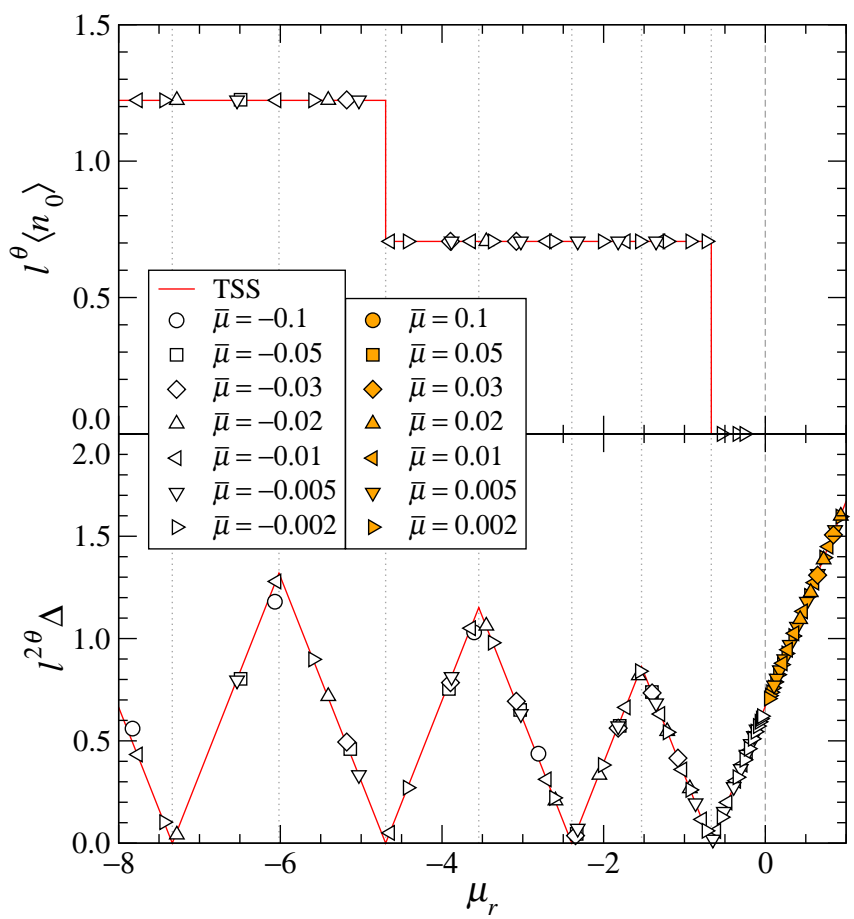

FIG. 2: The rescaled energy gap $l^{2 \theta} \Delta$ (below) and the rescaled particle density $l^{\theta}\left\langle n_{0}\right\rangle$ in the middle of the trap (above) for $p=4(\theta=3 / 4)$, compared with the predictions of TSS. We report results for several values of $\bar{\mu}$ and $l \gtrsim 10$. 


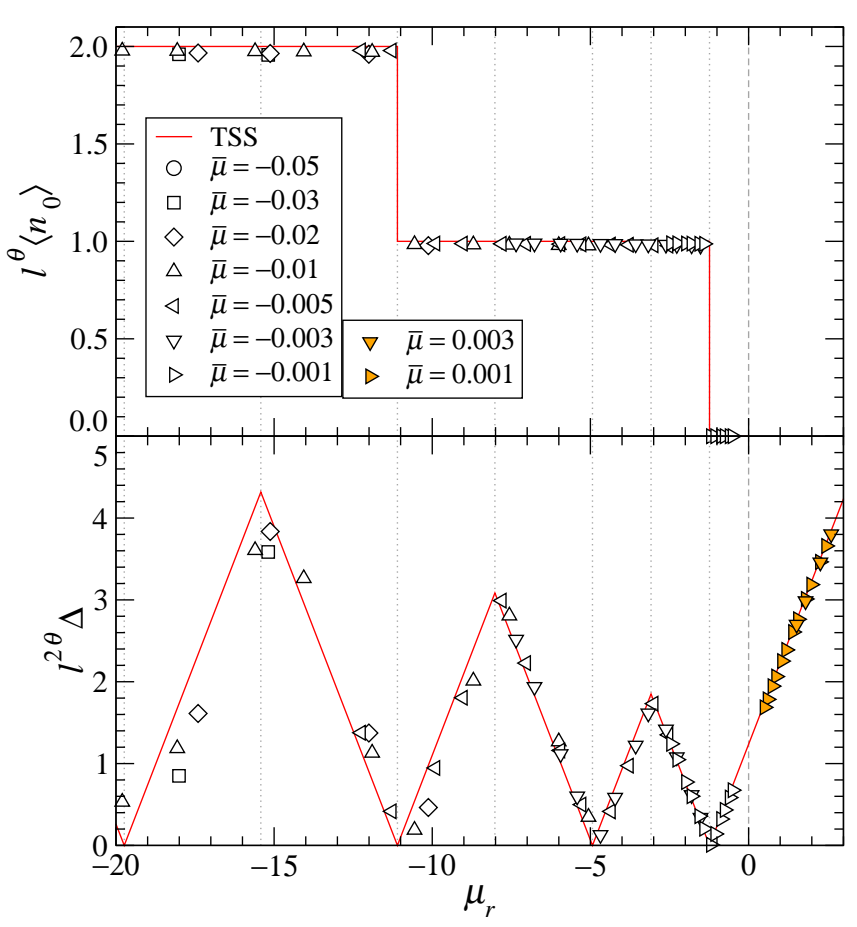

FIG. 3: The rescaled energy gap $l^{2 \theta} \Delta$ (below) and the rescaled particle density $l^{\theta}\left\langle n_{0}\right\rangle$ in the middle of the trap (above) for $p \rightarrow \infty(\theta=1)$, compared with the predictions of TSS. We report results for several values of $\bar{\mu}$ and $l \gtrsim 10$. They approach the analytical TSS results with increasing $l$.

function. Specifically, in the case of the gap $\Delta=E_{1}-E_{0}$ we have

$$
\Delta \approx l^{-2 \theta} \mathcal{E}_{\Delta}\left(\mu_{r}\right), \quad \mathcal{E}_{\Delta}\left(\mu_{r}\right)=\min _{k}\left|\Omega_{k}\right|,
$$

which can be easily computed from the solution of Eq. (15). The results are shown in Figs. 1, 2, and 3, respectively for $p=2,4$ and in the limit $p \rightarrow \infty$. For any $p$, the scaling function $\mathcal{E}_{\Delta}\left(\mu_{r}\right)$ shows a triangular structure for $\mu_{r} \leq 0$ and it is linear for $\mu_{r} \geq-\bar{\Omega}_{0}<0$. In Figs. 1,2 , and 3 we also show results obtained by numerical diagonalization at fixed $l$. They clearly approach the analytical TSS computations. Corrections to scaling turn out to be very small for $p=2$ and $p=4$.

\section{The particle density}

We now consider the expectation value and correlators of the particle density

$$
n_{x} \equiv b_{x}^{\dagger} b_{x}=\frac{1-\sigma_{x}^{z}}{2}=c_{x}^{\dagger} c_{x}=\sum_{k_{1}, k_{2}} \phi_{k_{1} x}^{*} \phi_{k_{2} x} \eta_{k_{1}}^{+} \eta_{k_{2}} .
$$

$\left\langle n_{x}\right\rangle$ is zero for $\bar{\mu} \geq 0$, but it can be nonzero for $\bar{\mu}<0$. Since the RG dimension of the particle density is given by $y_{n}=d+z-y_{\mu}=1$, we expect

$$
\left\langle n_{x}\right\rangle=l^{-\theta} \mathcal{D}\left(\mu_{r}, X\right)
$$

This is confirmed by the analytical results of the previous section, which lead to

$$
\mathcal{D}\left(\mu_{r}, X\right)=\sum_{k: \Omega_{k}<0} \varphi_{k}^{2}(X),
$$

where we used the fact that $\left\langle\eta_{k}^{+} \eta_{k}\right\rangle=1$ if $\Omega_{k}<0$ and zero otherwise. $\varphi_{k}(X)$ are the normalized eigenfunctions of Eq. (15). Note that $\mathcal{D}$ depends on $\mu_{r}$ only through the number of negative energy levels $N$, i.e., the number of levels included in the sum of Eq. (23); therefore, it vanishes for $\mu_{r} \geq-\bar{\Omega}_{0}$ and it falls on a discrete set of curves as a function of $X$ (with jumps at $\Omega_{k}=0$, i.e., zeroes of $\Delta)$. Since $\varphi_{k}(X)=(-1)^{k} \varphi_{k}(-X)$, only even $k$ s contribute to $\mathcal{D}\left(\mu_{r}, 0\right)$.

Specifically, for $p=2$ we obtain

$$
\begin{aligned}
& \mathcal{D}\left(\mu_{r}, 0\right)=2^{1 / 4} \pi^{-1 / 2} \sum_{j=0}^{j_{\max }} \frac{[(2 j-1) ! !]^{2}}{(2 j) !}, \\
& \left.j_{\max }=\max \left(0,|| \mu_{r} \mid / \sqrt{8}-1 / 4\right\rfloor\right),
\end{aligned}
$$

where $\lfloor x\rfloor \equiv$ floor $(x)$ is the largest integer not greater than $x$. For $p \rightarrow \infty$ we have

$$
\mathcal{D}\left(\mu_{r}, 0\right)=\max \left(0,\left\lfloor\sqrt{2\left|\mu_{r}\right|} / \pi+1 / 2\right\rfloor\right) .
$$

Numerical results for the particle density at the origin are shown in Figs. 1, 2, and 3, for $p=2,4, \infty$ respectively; they fully support TSS. Note the peculiar plateaus and the discontinuities in the particle density at negative values of the scaling variable $\mu_{r}$. Moreover, asymptotically for $\mu_{r} \rightarrow-\infty,\left\langle n_{0}\right\rangle \sim|\bar{\mu}|^{1 / 2}$, which matches the critical behavior for $\bar{\mu}<0$ in the absence of the trap [25]. Numerical results for $\left\langle n_{x}\right\rangle$, showing the dependence on the distance form the middle of the trap, are shown in Fig. 4, for $-0.01 \leq \bar{\mu}<0$ and $l \geq 10$. They show that the quantity $l^{\theta}\left\langle n_{x}\right\rangle$ approaches the analytical functions obtained using Eq. (23).

\section{The particle density correlator}

Let us consider the particle density correlation

$$
G_{n}(x) \equiv\left\langle n_{0} n_{x}\right\rangle-\left\langle n_{0}\right\rangle\left\langle n_{x}\right\rangle=\frac{1}{4}\left\langle\sigma_{0}^{z} \sigma_{x}^{z}\right\rangle_{c} .
$$

Like the particle density, it vanishes for $\bar{\mu} \geq 0$, and it can be nonzero for $\bar{\mu}<0$. In the TSS limit we expect the scaling behavior

$$
G_{n}(x) \approx l^{-2 \theta} \mathcal{G}_{n}\left(\mu_{r}, X\right) .
$$

Straightforward calculations, i.e., writing $G_{n}$ in terms of $\eta$-operators and then using the Wick theorem to compute the resulting ground-state expectation values, show that 

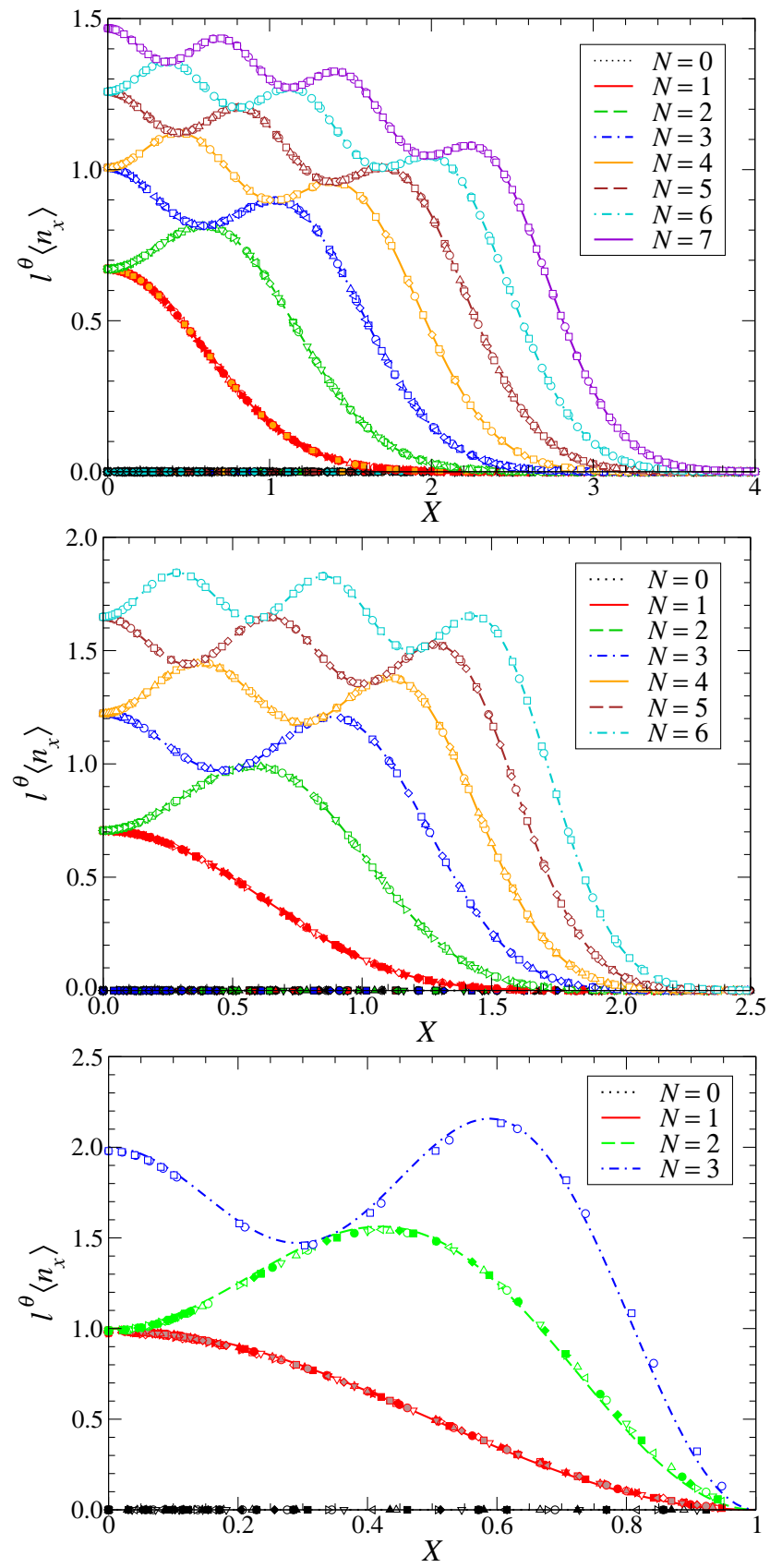

FIG. 4: The rescaled density $l^{\theta}\left\langle n_{x}\right\rangle$ for $p=2$ (above), $p=4$ (middle), and $p \rightarrow \infty$ (below), compared with Eq. (23). $N$ is the number of negative energy levels with $\Omega_{k}<0$. Here and in the following, set of parameters $(\mu, l)$ are differentiated by symbol color (matching $N$ ), shape, and filling color. The results approach the analytical $N$-dependent TSS results, cf. Eq. (23), with increasing $l$.

the scaling function $\mathcal{G}_{n}\left(\mu_{r}, X\right)$ can be written in terms of the eigensolutions of Eq. (15):

$$
\begin{aligned}
& \mathcal{G}_{n}\left(\mu_{r}, X\right)= \\
& \quad\left[\sum_{k: \Omega_{k}<0} \varphi_{k}(X) \varphi_{k}(0)\right]\left[\sum_{k: \Omega_{k}>0} \varphi_{k}(X) \varphi_{k}(0)\right]
\end{aligned}
$$

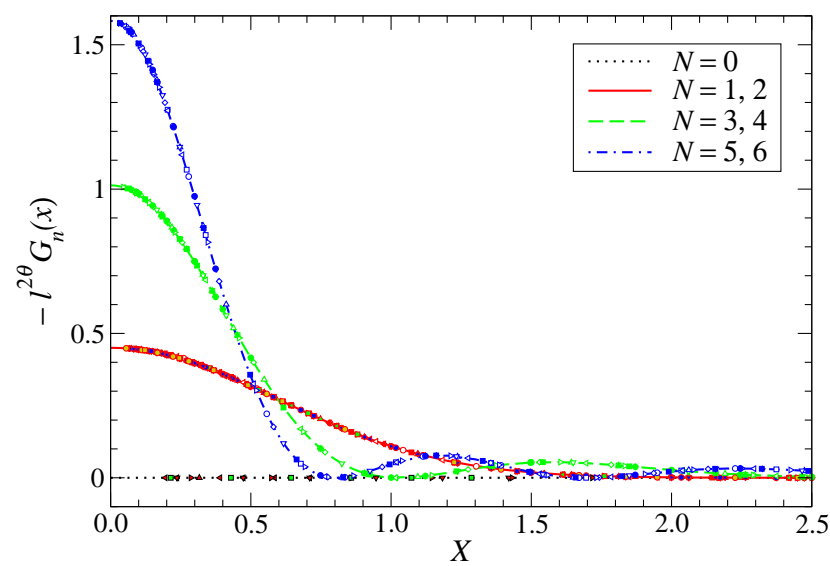

FIG. 5: The rescaled connected correlation $l^{2 \theta} G_{n}(x)$ for $p=$ $2, l \geq 10, \mu \geq 0.95$, and $N \leq 6$. The results approach the analytical $N$-dependent TSS results, cf. Eq. (29), with increasing $l$.

then, using the completeness relation $\sum_{k} \varphi_{k}(X) \varphi_{k}(0)=$ $\delta(X)$, we obtain

$$
\mathcal{G}_{n}\left(\mu_{r}, X\right)=-\left[\sum_{k: \Omega_{k}<0} \varphi_{k}(X) \varphi_{k}(0)\right]^{2} ;
$$

note that odd $k \mathrm{~s}$ do not contribute to the sum. Like the particle density, $\mathcal{G}_{n}\left(\mu_{r}, X\right)$ is nonzero only for $\mu_{r}<$ $-\bar{\Omega}_{0}<0$, and depends on $\mu_{r}$ only through $N$ (actually, only through $\lfloor(N+1) / 2\rfloor)$.

From the numerical data, we find that $l^{2 \theta} G_{n}(x)$ as a function of $X$ approaches rapidly $\mathcal{G}_{n}\left(\mu_{r}, X\right)$. The results for $p=2$ are shown in Fig. 5.

\section{The one-particle density matrix}

We now consider the one-particle density matrix defined as

$$
G_{b}\left(x_{i}, x_{j}\right) \equiv\left\langle b_{i}^{\dagger} b_{j}\right\rangle=\left\langle\sigma_{i}^{-} \sigma_{j}^{+}\right\rangle,
$$

where $\sigma^{ \pm}=\left(\sigma^{x} \pm i \sigma^{y}\right) / 2$. For $\bar{\mu} \geq 0$, since the ground state is empty, $G_{b}\left(x_{i}, x_{j}\right)=0$. But, like the particle density (note that $G_{b}(x, x)=\left\langle n_{x}\right\rangle$ ), it can be nonzero for $\bar{\mu}<0$. Its scaling behavior is also determined by the RG dimension $y_{b}$ of the field associated with the boson operator $b_{i}$, which is [25] $y_{b}=1 / 2$. Considering specifically the correlations with one boson operator at the origin, i.e., $G_{b}(x, 0)$, we expect

$$
G_{b}(x, 0) \approx l^{-\theta} \mathcal{M}\left(\mu_{r}, X\right) .
$$

Numerical results can be obtained using the method of Ref. [27]. They confirm the scaling behavior (31), i.e., $l^{\theta} G_{b}(x, 0)$ appears to approach a function of $\mu_{r}$ and $X$ in the large-trap limit. Fig. 6 shows results for $p=2$. Again, the dependence on $\mu_{r}$ is only through the number $N$ of negative energy levels. 


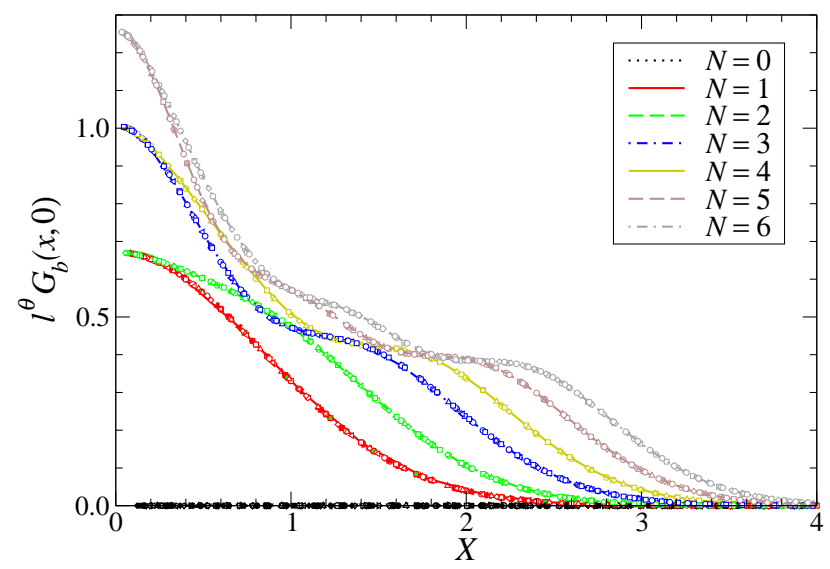

FIG. 6: The rescaled $\sigma_{x}$ correlation $l^{\theta} G_{b}(x, 0)$ for $p=2$, $l \geq 10, \mu \geq 0.95$, and $N \leq 6$. With increasing $l$, they converge toward $N$-dependent TSS curves.

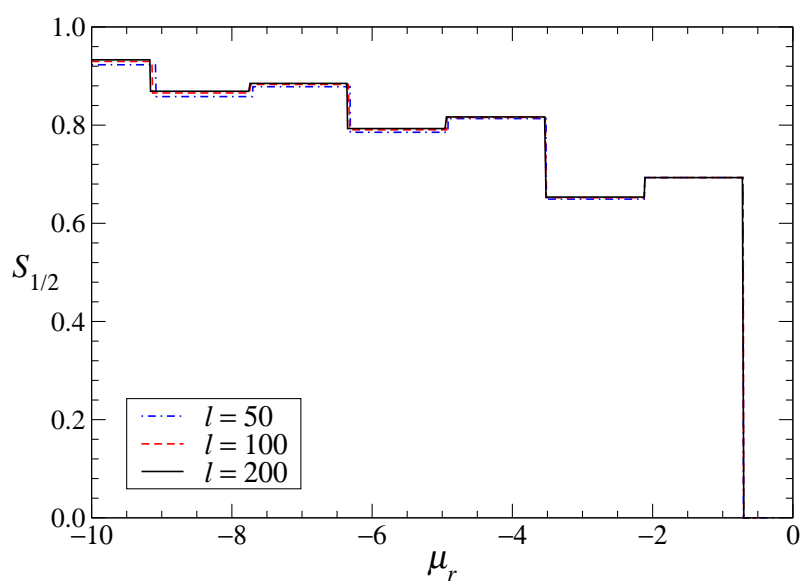

FIG. 7: The half-lattice von Neumann entanglement entropy $S_{1 / 2}$, cf. Eq. (32), for $p=2$.

\section{The von Neumann entanglement entropy}

We consider the von Neumann entanglement entropy $S\left(l_{A} ; L\right)$ in the presence of the confining potential, defined by dividing the chain in two parts of length $l_{A}$ and $L-l_{A}$. The entanglement entropy trivially vanishes for $\bar{\mu} \geq 0$, but it can be nonzero for $\bar{\mu}<0$ [28]. It can be computed using the techniques of Ref. [29]. We consider the half-lattice von Neumann entanglement entropy $S(L / 2 ; L)$ for even $L$ and open boundary conditions in the presence of the trap of size $l$ (in this case the trap is centered between the two central sites of the chains). Its large- $L$ limit,

$$
S_{1 / 2} \equiv \lim _{L \rightarrow \infty} S(L / 2 ; L)
$$

depends on the trap size $l$ only. The TSS limit of $S_{1 / 2}$ turns out to depend only on $N$, and therefore it is a function of the scaling quantity $\mu_{r}$, as shown in Fig. 7 for the harmonic potential.

\section{Universality of the TSS}

In order to check the universality of the TSS, we consider the XXZ chain model

$$
\begin{aligned}
H_{\mathrm{XXZ}}= & -\sum_{i=1}\left(S_{i}^{x} S_{i+1}^{x}+S_{i}^{y} S_{i+1}^{y}+j_{z} S_{i}^{z} S_{i+1}^{z}\right) \\
& -\sum_{i=1}\left[\mu+V\left(x_{i}\right)\right] S_{i}^{z}
\end{aligned}
$$

In terms of bosonic operators $b_{i}$, the $j_{z}$ term corresponds to nearest-neighbor density-density interactions, i.e.,

$$
j_{z} \sum_{i}\left(n_{i}-1 / 2\right)\left(n_{i+1}-1 / 2\right)
$$

where $n_{i}=b_{i}^{\dagger} b_{i}$.

For $\left|j_{z}\right|<1$, the homogeneous XXZ chain model (i.e., with $V=0$ ) undergoes two Mott insulator to superfluid transitions at $\mu_{c}^{ \pm}= \pm\left(1+j_{z}\right)$ in the same universality class of those of the XX model at $\mu= \pm 1$. Indeed, the $j_{z}$ term is irrelevant at these transitions: the RG dimension of the coupling $j_{z}$ is $y_{j_{z}}=-1$ [25]. We therefore expect that the $j_{z}$ term remains irrelevant also in the TSS limit. The main difference concerns the dominant scaling corrections, which are expected to be $O\left(\xi^{-1}\right)$, thus $O\left(l^{-\theta}\right)$, when this term is present. Therefore they are expected to be larger than those of the XX model, where they are $O\left(l^{-2 \theta}\right)$.

The Hamiltonian (33) is no longer equivalent to a quadratic fermionic Hamiltonian. The properties of the model can be studied numerically via DMRG. Results for the gap and the particle density in the middle of the trap at the low-density Mott transition, around $\mu_{c}^{+}$(we define $\bar{\mu}=\mu-\mu_{c}^{+}$), are shown in Fig. 8 for $p=2$. They clearly support universality of TSS. As expected, corrections to scaling are larger for $j_{z} \neq 0$ than for $j_{z}=0$.

It is interesting to notice that energy differences and expectation values over states with $z$-component of the total spin $M=L / 2$ and $M=L / 2-1$ depend on $\mu$ and $j_{z}$ only through $\mu_{c}^{+}$. This implies that, in the scaling region, vacuum expectation values (as functions of $\mu_{r}$ ) are independent on $j_{z}$ for $\mu_{r} \gtrsim-\bar{\Omega}_{2}$; the energy gap $\Delta$ is independent on $j_{z}$ for $\mu_{r} \gtrsim-\left(\bar{\Omega}_{1}+\bar{\Omega}_{2}\right) / 2$. This is clearly shown in Fig. 8.

\section{THE LOCAL DENSITY APPROXIMATION OF THE PARTICLE DENSITY}

The homogeneous 1D hard-core $\mathrm{BH}$ model, i.e., the model (1) for $U \rightarrow \infty$ and $V(x)=0$, has a nonzero filling below the low-density Mott transition, i.e., for $\mu<1$. In the infinite-chain limit $L \rightarrow \infty$, the filling $f$ for $|\mu| \leq 1$ is given by [25]

$$
f \equiv\left\langle n_{i}\right\rangle=\frac{N}{L}=\frac{1}{\pi} \arccos \mu, \quad \mu=\cos \pi f .
$$



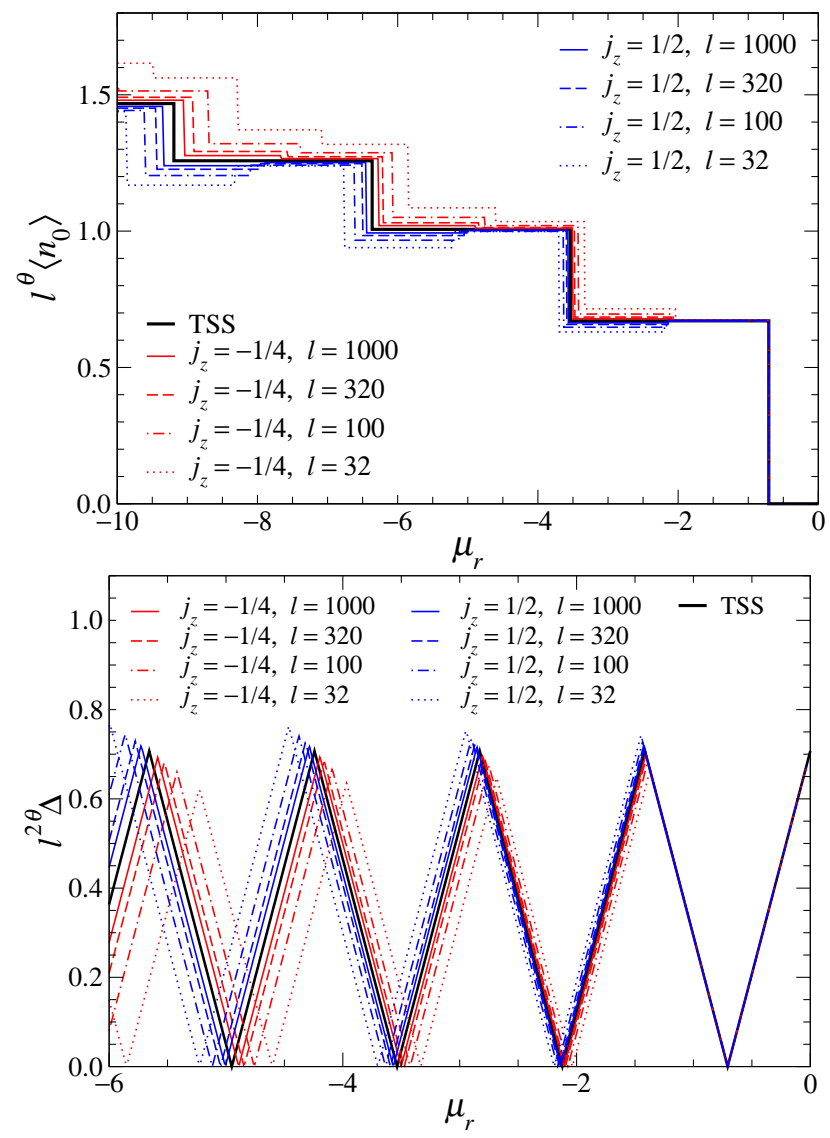

FIG. 8: Scaling plot of the energy gap (below) and of the particle density at the origin (above) for the XXZ model with $p=2$ and $j_{z}=-1 / 4,1 / 2$. The data at $j_{z}=-1 / 4$ and $1 / 2$ approach the TSS functions, with increasing $l$, from opposite sides.

The corresponding Fermi momentum is $k_{F}=\pi f$. For $\mu \leq-1, f=1$ independently of $\mu$. In the following, $f$ will always denote the value for the infinite homogeneous chain.

In the presence of a space-dependent confining potential, the so-called local density approximation (LDA) estimates the spatial dependence of the particle density by taking the value of the particle density of the homogeneous system at the effective chemical potential

$$
\mu_{\mathrm{eff}}(x) \equiv \mu+(x / l)^{p} .
$$

The LDA has been widely used to get quantitative information on the behavior of $\mathrm{BH}$ models in a confining potential, and, more generally, of inhomogeneous systems, see, e.g., Refs. [13, 16, 17, 30, 31].

The LDA of the particle density reads

$$
\begin{aligned}
& \left\langle n_{x}\right\rangle_{\text {lda }} \equiv \rho_{\text {lda }}(x / l)= \\
& \begin{cases}0 & \text { for } \mu_{\mathrm{eff}}(x)>1, \\
(1 / \pi) \arccos \mu_{\mathrm{eff}}(x) & \text { for }-1 \leq \mu_{\mathrm{eff}}(x) \leq 1, \\
1 & \text { for } \mu_{\mathrm{eff}}(x)<-1\end{cases}
\end{aligned}
$$

This would imply the presence of a plateau at $n=1$

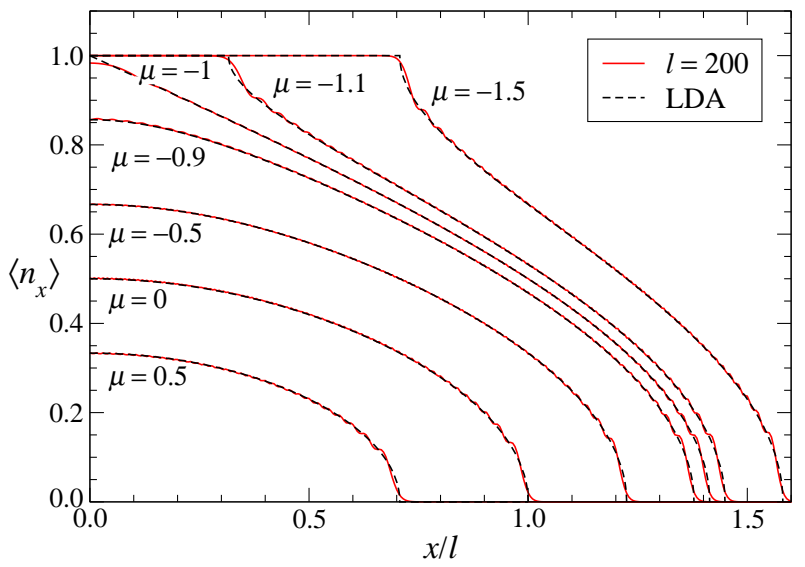

FIG. 9: The particle density vs. $x / l$ for $p=2$ and several values of $\mu$. We show results from the LDA, cf. Eq. (37), and from numerical calculations on a large chain with $l=200$. The differences are hardly visible in the figure.

when $\mu_{\mathrm{eff}} \leq-1$, for

$$
x / l \leq(-1-\mu)^{1 / p},
$$

and a vanishing particle density when $\mu_{\mathrm{eff}} \geq 1$, for

$$
x / l \geq(1-\mu)^{1 / p} .
$$

In Fig. 9 we compare the LDA of the particle density with numerical results for $p=2$ and the trap size $l=200$. Note the flat regions related to the $n=0,1$ Mott phases, already observed in experimental and numerical works, see, e.g., Refs. [7, 10, 13]. Analogous results are found for other powers of the confining potential. The LDA provides a good approximation of the particle density, which improves with increasing trap size. The differences of the trap-size dependence from the LDA results show a nontrivial scaling behavior; they will be considered in the next sections.

Using Eq. (37) we can also obtain the LDA of the total particle number:

$$
N_{\mathrm{lda}}=\sum_{x}\left\langle n_{x}\right\rangle_{\mathrm{lda}} \approx 2 l \int_{0}^{\infty} \rho_{\mathrm{lda}}(y) d y \equiv c(\mu) l,
$$

where $c(\mu)$ is a finite function of $\mu$, which can be easily computed by integrating $\rho_{\text {lda }}(y)$. Comparing with the numerical results, we find excellent agreement, i.e.,

$$
\left|c(\mu) l-N_{\text {exact }}\right|<1
$$

(note that the $N_{\text {exact }}$ is an integer number, while the LDA is a continuous linear function). Eq. (41) implies that, in the large- $l$ limit at fixed $\mu$, the total particle number increases as

$$
N \sim l
$$




\section{TRAP-SIZE DEPENDENCE IN THE SUPERFLUID PHASE}

We now discuss the trap-size dependence in the gapless superfluid phase for $|\mu|<1$. In the continuum limit, the gapless superfluid phase of the homogeneous system is described by a free massless bosonic field theory with dynamic exponent $z=1$, corresponding to a conformal field theory with central charge $c=1$, see, e.g., Ref. [26].

\section{A. Level crossings in the presence of the trap}

In the gapless superfluid phase, and more generally for $\bar{\mu} \equiv \mu-1<0$, the ground state contains all the $\eta$ fermions with $\omega_{k}<0$, cf. Eq. (10). In the presence of the trapping potential (2), level crossings of the lowest states occur in the $\bar{\mu}-l$ plane separating the regions with $N=k$ and $N=k+1$. This is essentially related to the fact that the particle number is conserved, i.e., the particle number operator $\hat{N}=\sum_{i} b_{i}^{\dagger} b_{i}$ commutes with the $\mathrm{BH}$ Hamiltonian (1); thus the eigenvectors do not depend on $\mu$, even though the eigenvalues do. Since, for $\mu<1$ and in the absence of the trap potential, the ground state has a finite density $N / L>0$, and in the presence of the trap $N$ is finite and increases as $N \sim l$, the lowest states show an infinite number of level crossings as $l \rightarrow \infty$ (after $L \rightarrow \infty$ ) where the gap $\Delta$ vanishes. Note that the hardcore limit, $U \rightarrow \infty$ in Eq. (1), does not play any special role, so we expect that level crossings at finite trap size are a general feature of the $\mathrm{BH}$ model in the presence of a confining potential, when the homogeneous limit of infinite trap size has a finite particle density.

In the following of this section we present results for the trap-size dependence of several observables in the superfluid phase, for $|\mu|<1$. As we shall see, the abovementioned level crossings and the competition of smooth modes and modes at the Fermi momentum $k_{F}=\pi f$ give rise to peculiar modulated trap-size dependencies.

\section{B. Analytical results for the energy gap at small $|\bar{\mu}|$}

We can infer some information on the trap-size dependence of the energy gap at $\bar{\mu}<0$ and small $|\bar{\mu}|$ by using the analytical calculations of Sec. II A. Let us introduce a few definitions. $E_{0}^{(k)}(l)$ is the lowest energy in the sector with $N=k . l_{0}^{(k)}$ is the value of $l$ such that $E_{0}^{(k)}(l)=E_{0}^{(k+1)}(l)$; Eq. (10) implies that this is also the doubly-degenerate ground-state energy. The gap shows peaks at $l_{\text {peak }}^{(k)}$ with $l_{0}^{(k)}<l_{\text {peak }}^{(k)}<l_{0}^{(k+1)} ; l_{\text {peak }}^{(k)}$ is the value of $l$ such that $E_{0}^{(k-1)}(l)=E_{0}^{(k+1)}(l)$; this is the energy of the doubly-degenerate first excited state in the sector with $N=k$, where the energy of the ground state is $E_{0}^{(k)}$.

For $p=2$ Eq. (16) tells us that, for large trap size, the gap vanishes at

$$
l_{0}^{(k)} \approx \frac{\sqrt{2}}{|\bar{\mu}|}(k+1 / 2)
$$

and therefore the interval between two zeroes is constant, i.e.,

$$
l_{0}^{(k+1)}-l_{0}^{(k)} \approx \frac{\sqrt{2}}{|\bar{\mu}|} .
$$

Moreover, the gap $\Delta=\min _{k}\left|\omega_{k}\right|$ has peaks at

$$
l_{\text {peak }}^{(k)} \approx \frac{\sqrt{2}}{|\bar{\mu}|}(k+1),
$$

where the gap decreases as

$$
\Delta_{\text {peak }} \sim l^{-1} .
$$

The product between the trap size and the gap, i.e., $l \Delta$, has a periodic asymptotic behavior. Indeed, defining

$$
\phi=\frac{l-l_{0}^{(k)}}{l_{0}^{(k+1)}-l_{0}^{(k)}}, \quad \text { for } \quad l_{0}^{(k)} \leq l<l_{0}^{(k+1)},
$$

thus $0 \leq \phi<1$, we obtain a simple triangle-like form

$$
l \Delta=\operatorname{at}(\phi)
$$

where $t(\phi)$ is the triangle function

$$
t(\phi)=1 / 2-|\phi-1 / 2|
$$

and $a=\sqrt{2}$. Note that $l_{\text {peak }}^{(k)}$ corresponds asymptotically to the value $\phi=1 / 2$.

More generally, for any $p$, the asymptotic behavior (18) for large $k$ of the eigensolutions of Eq. (15) implies

$$
l_{0}^{(k)} \sim k|\bar{\mu}|^{-\rho}, \quad \rho=\frac{p+2}{2 p} .
$$

Thus, for any power $p$, the location of the zeroes increases linearly with $k$, and therefore the intervals between subsequent zeroes $l_{0}^{(k+1)}-l_{0}^{(k)}$ approaches a constant as in the $p=2$ case. This implies again that the peak of the gap decreases as $l^{-1}$, and that the product $l \Delta$ is a periodic function of $l$, and, specifically, of the corresponding phase-like variable $\phi$, cf. Eq. (47). Moreover, asymptotically, $l \Delta$ must have a triangle-like form for any $p$, analogous to Eq. (48). Notable values are

$$
\begin{array}{cl}
l_{0}^{(k)} \approx \gamma_{4}|\bar{\mu}|^{-3 / 4}(k+1 / 2) & (p=4), \\
l_{0}^{(k)} \approx \pi 2^{-3 / 2}|\bar{\mu}|^{-1 / 2}(k+1) & (p=\infty),
\end{array}
$$

and

$$
\begin{aligned}
& a=\frac{4}{3} \gamma_{4}|\bar{\mu}|^{1 / 4} \quad(p=4), \\
& a=\pi \sqrt{|\bar{\mu}| / 2} \quad(p=\infty) .
\end{aligned}
$$

The above results suggest that the gap vanishes with a global power scaling $\Delta \sim l^{-1}$. However, its amplitude in not a constant, but a periodic function of the trap size. This scenario will be confirmed by the results from numerical diagonalization at fixed $\mu<1$. 
TABLE I: Numerical results in the superfluid region for the XX model and several values of $\mu$ and $p$, for the asymptotic interval $Q_{l}^{*}$ between level crossings, the constant $a$ entering the asymptotic behavior (56) of the gap, the constant $b$ appearing in Eq. (59) of the particle density in the middle of trap, and the amplitude of the entanglement length scale $a_{e}$, cf. Eq. (70). The reported estimates are obtained by extrapolating data at finite trap size; their uncertainty is at most one on the last figure.

\begin{tabular}{ccclll}
\hline \hline$\mu$ & $p$ & $Q_{l}^{*}$ & \multicolumn{1}{c}{$a$} & \multicolumn{1}{c}{$b$} & \multicolumn{1}{c}{$a_{e}$} \\
\hline$-1 / 2$ & 2 & 0.8265187 & 1.03010681 & 0.378618 & 2.64118 \\
$-1 / 2$ & 4 & 0.8021659 & 1.19898955 & 0.440691 & 2.26916 \\
0 & 2 & 1.3110287 & 1.198140234 & 0.3813798 & 2.622058 \\
0 & 4 & 1.1635926 & 1.349953898 & 0.4297036 & 2.327185 \\
$1 / 2$ & 2 & 2.7337528 & 1.3177760 & 0.484352 & 2.06462 \\
$1 / 2$ & 4 & 2.0551051 & 1.29539790 & 0.476126 & 2.10028 \\
\hline \hline
\end{tabular}

\section{Results from numerical diagonalization}

We now present numerical results at fixed $l$ and $\mu$, obtained by numerical diagonalization of the quadratic Hamiltonian (8), see App. A. The results at fixed trap size are essentially correct up to machine precision.

\section{Interval between level crossings}

The numerical results show that the interval between two level crossing approaches a constant value in the large trap-size limit. They turn out to fit the simple Ansatz

$$
Q_{l}^{(k)} \equiv l_{0}^{(k+1)}-l_{0}^{(k)}=Q_{l}^{*}+c_{2} l^{-2}+c_{3} l^{-3}+\ldots,
$$

for sufficiently large trap size, $l \gtrsim 10$, which allows us to get accurate estimates of the large- $l$ limit $Q_{l}^{*}$. We report a selection of results in Table I.

Note that the results for $p=2$ are quite close to, and clearly approach, the small- $\bar{\mu}$ estimate (44), i.e., $Q_{l}^{*} \approx$ $\sqrt{2} /|\bar{\mu}|$. In the limit $p \rightarrow \infty$ the value of $Q_{l}^{*}$ should converge toward the corresponding value in the finite-size behavior of the homogeneous system with open boundary conditions, see App. B. Thus

$$
\lim _{p \rightarrow \infty} Q_{l}^{*}=\frac{1}{2 f}
$$

where $f$ is the filling factor given in Eq. (35).

The asymptotic values $Q_{l}^{*}$ can also be computed using the LDA of the total particle number, cf. Eq. (40). Since the level crossings occur at the boundary between the regions with $N=k$ and $N=k+1$, asymptotically we have $k \approx N_{\text {lda }}=c(\mu) l$, thus

$$
Q_{l}^{*}=1 / c(\mu)
$$

whose numerical values coincide with the estimates of $Q_{l}^{*}$ obtained above, see Table I. This is actually a further evidence, beside the results of Sect. III, that the LDA of the particle number is asymptotically exact.

\section{Trap-size dependence of the gap}

For any $|\mu|<1$ and power of the confining potential, the results for the gap show the behavior

$$
\begin{aligned}
& \Delta=l^{-1} A_{\Delta}(\phi)+O\left(l^{-2}\right), \\
& A_{\Delta}(\phi)=a t(\phi),
\end{aligned}
$$

with $t(\phi)$ given by Eq. (49), and the coefficient $a$ depends on $p$ and $\mu$. We determine the single parameter $a$ by fitting the peak values to the form

$$
\Delta_{\text {peak }}=\frac{1}{2} a l^{-1}+c l^{-3}
$$

(the term $l^{-2}$ is absent just for this quantity); the fit quality is usually excellent for $l \gtrsim 10$. We report some results for the constant $a$ in Table I. Note that the values for $p=2$ appear to approach the small- $|\bar{\mu}|$ estimate $a=$ $\sqrt{2}$ with increasing $\mu$. The value of $\phi$ at the peak of the gap converges as $\phi_{\text {peak }}=1 / 2+c l^{-1}+O\left(l^{-2}\right)$ with $c \ll 1$, of the order of $10^{-2}$.

We note that the modulated trap-size dependence of the gap within the superfluid region $|\mu|<1$ appears to be largely universal, being substantially independent of $\mu$ and $p$, apart from a trivial normalization.

This is also confirmed by the finite-size behavior of the homogeneous XX chain corresponding to the $p \rightarrow \infty$ limit of the confining potential, where the model becomes equivalent to a homogeneous chain of size $L=2 l$ with open boundary conditions (more precisely, the $p \rightarrow \infty$ limit corresponds to a chain with $L=2\lfloor l\rfloor+1$ when the center of the trap coincides with the middle site of the chain, and $L=2\lfloor l\rfloor$ when the center is in the middle between two sites). The results of App. B show that the modulated trap-size behavior of the gap found at finite values of $p$ persists in the $p=\infty$ limit.

\section{The particle density}

The particle density $\left\langle n_{0}\right\rangle$ at the origin shows a modulated asymptotic behavior as well, but with a period twice the period of $\Delta$; therefore it is useful to define

$$
\bar{\phi}=2 \frac{l-l_{0}^{(2 k)}}{l_{0}^{(2 k+2)}-l_{0}^{(2 k)}} \quad \text { for } l_{0}^{(2 k)} \leq l<l_{0}^{(2 k+2)} ;
$$

thus $0 \leq \bar{\phi}<2$ (note that either $\bar{\phi}=\phi$ or $\bar{\phi}=\phi+1$ for large $l$ ). The results of the numerical diagonalization show that $\left\langle n_{0}\right\rangle$ satisfies the asymptotic behavior

$$
\begin{aligned}
& \left\langle n_{0}\right\rangle-f=l^{-1} A_{n}(\bar{\phi})+O\left(l^{-2}\right), \\
& A_{n}(\bar{\phi})=b(1-\bar{\phi}),
\end{aligned}
$$



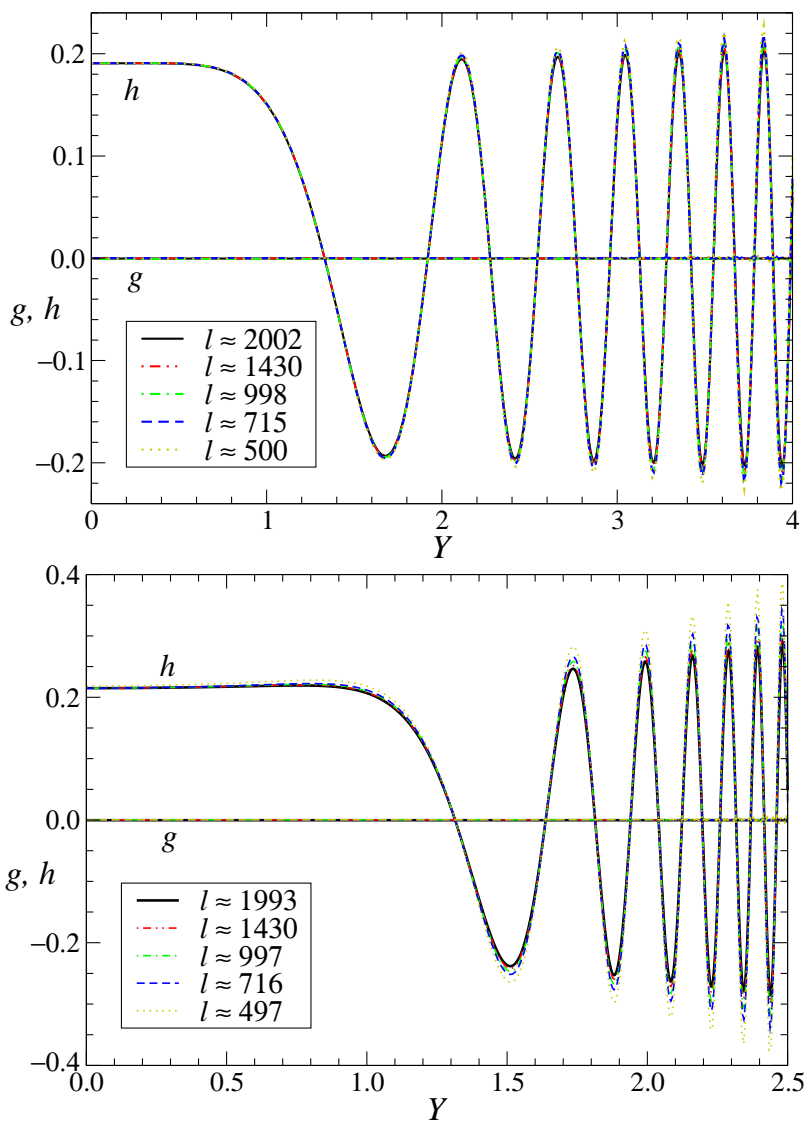

FIG. 10: The functions $h$ and $g$, cf. Eq. (60), vs. $Y=$ $l^{-p /(p+1)} x$ at odd peaks of $\Delta$ at $\mu=0$, for $p=2$ (above) and $p=4$ (below). Results for $p=6$ are of the same quality. $h$ is real, since $k_{F}=\pi / 2 . g$ at peaks is compatible with $0 . h$ at even peaks is opposite in sign to $h$ at odd peaks. The data at different trap sizes clearly approach TSS functions when increasing $l$. Here and in the following, in the legends $l$ is rounded to the nearest integer for the sake of presentation.

where $f$ is the filling factor of the homogeneous model without trap, cf. Eq. (35). Some results for the constant $b$ are reported in Table I. Note that the $O\left(l^{-1}\right)$ term has zero average over the period.

An analogous behavior of the particle density in the middle of trap is found in the limit $p \rightarrow \infty$, as shown by the results of App. B for the finite-size behavior of the homogeneous XX chain with open boundary conditions.

The particle density as a function of the distance $x$ from the middle of the trap turns out to behave as

$$
\begin{aligned}
& \left\langle n_{x}\right\rangle \approx \rho_{\mathrm{lda}}(X)+l^{-1} \operatorname{Re}\left\{h(Y, \bar{\phi}) e^{2 i k_{F} x}+g(Y, \bar{\phi})\right\} \\
& X=x / l, \quad Y=x l^{-p /(p+1)},
\end{aligned}
$$

where $k_{F}=\pi f=\arccos \mu, g$ is real, and terms suppressed by higher powers of $l^{-1}$ are neglected. $g$ and $h$ are discontinuous at $\bar{\phi}=1$. Note that two scaling variables $X$ and $Y$ appear in the above equation, distinguishing the scaling behavior of the two terms. Note also that the leading term depending on $X$ is the LDA of the

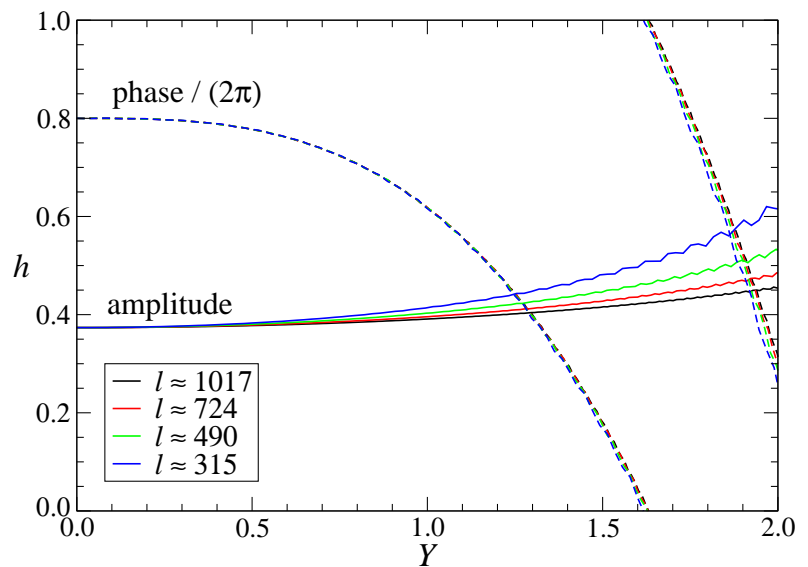

FIG. 11: The functions $h$ (solid lines) and $g$ (dashed lines), cf. Eq. (60), vs. $Y=l^{-2 / 3} x$ at odd peaks of $\Delta$ for $p=2$ and $\mu=$ $\cos \pi / 5(f=1 / 5) . g$ at peaks is compatible with $0 . h$ at even peaks is opposite in sign to $h$ at odd peaks. The "noise" more evident at smaller $l$ is due to the ambiguity of disentangling a "fast" oscillation $e^{2 i k_{F} x}$ from a "slow" oscillation $h(Y) /|h(Y)|$ in Eq. (60).

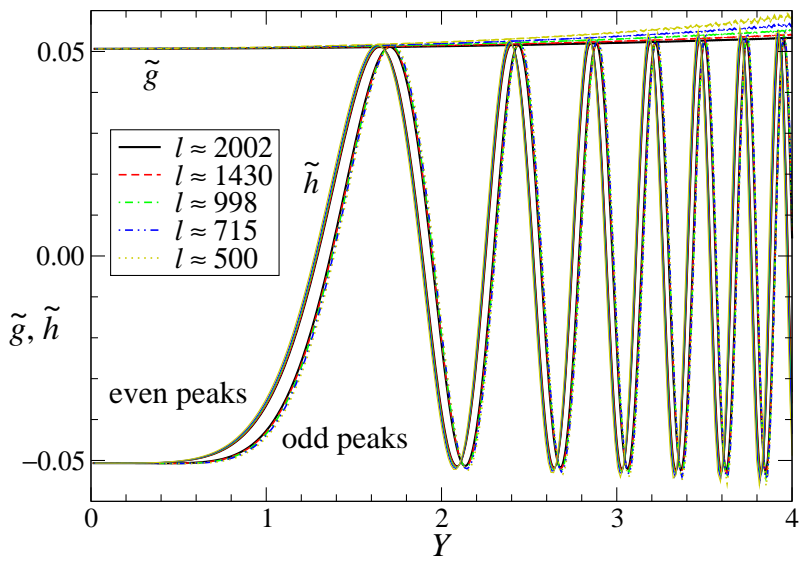

FIG. 12: The functions $\tilde{h}$ and $\tilde{g}$, cf. Eq. (61), vs. $Y=l^{-2 / 3} x$ at peaks of $\Delta$ for $p=2$ and $\mu=0 ; \tilde{h}$ is real, since $k_{F}=\pi / 2$. The data at different trap sizes clearly approach TSS functions when increasing $l$.

particle density, cf. Eq. (37). Some results are plotted in Figs. 10 and 11. We find that $g(Y)=0$ at peaks of $\Delta$ $(\phi=1 / 2,3 / 2)$.

\section{The particle density correlation}

Results for $p=2,4$, and 6 show also that the connected density correlation scales as

$$
G_{n}(x, 0) \approx l^{-2 p /(p+1)} \operatorname{Re}\left\{\tilde{h}(Y, \bar{\phi}) e^{2 i k_{F} x}+\tilde{g}(Y, \bar{\phi})\right\},
$$

where $Y=x l^{-p /(p+1)}$, and $\tilde{g}$ is real. $\tilde{h}$ and $\tilde{g}$ are discontinuous at $\bar{\phi}=1$. Results are shown in Figs. 12 and 13 at $\mu=0$ and $p=2,4$ respectively, and in Fig. 14 at 


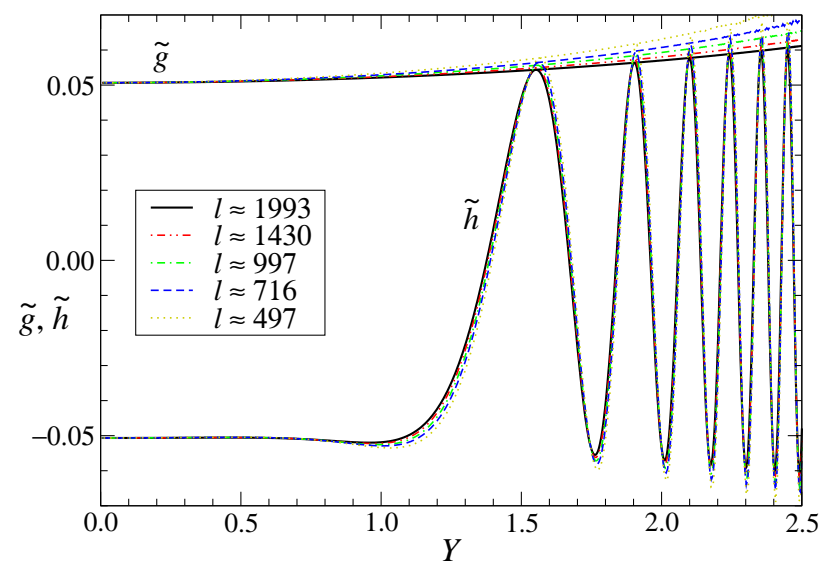

FIG. 13: The functions $\tilde{h}$ (solid lines) and $\tilde{g}$ (dashed lines), cf. Eq. (61), vs. $Y=l^{-p /(p+1)} x$ at $\mu=0$ and at odd peaks of $\Delta$ for $p=4$. Results for $p=6$ are of the same quality.

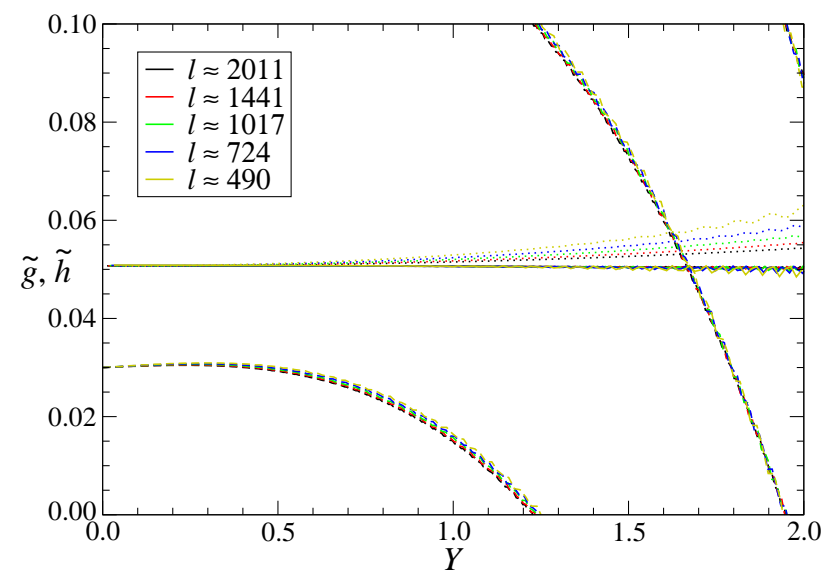

FIG. 14: The functions $\tilde{h}$ (solid lines: amplitude; dashed lines: phase $/(20 \pi))$ and $\tilde{g}$ (dotted lines) vs. $Y=l^{-2 / 3} x$ at odd peaks of $\Delta$ for $p=2$ and $\mu=\cos \pi / 5(f=1 / 5)$.

$\mu=\cos \pi / 5$ and $p=2$, at the peaks of the gap. We find that $\tilde{g}(Y)=|\tilde{h}(Y)|$ at peaks of $\Delta(\phi=1 / 2,3 / 2)$.

\section{The one-particle density matrix}

Another interesting quantity is the one-particle density matrix (30). In the homogeneous system without trap

$$
G_{b}\left(x_{i}, x_{j}\right) \sim\left|x_{i}-x_{j}\right|^{-1 / 2}
$$

from which we can read the RG dimension of the bosonic operator $b$ in the superfluid phase, i.e., $y_{b}=1 / 4$. This behavior is also observed in the presence of the trap for sufficiently small distances [18]. Fig. 15 shows results for $p=2$ and $\mu=0$ : the data of $x^{1 / 2} G_{b}(x, 0)$ for different $l \mathrm{~s}$ appear to collapse to a unique curve when plotted versus $x / l$, apart from small oscillations with a decreasing amplitude for $l \rightarrow \infty$. Only the oscillations depend on $\phi$.

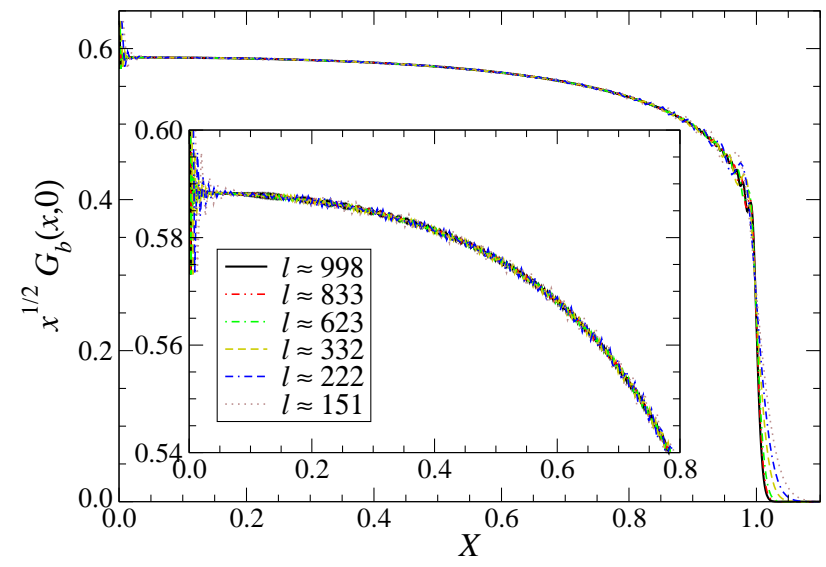

FIG. 15: $x^{1 / 2} G_{b}(x, 0)$ at peaks of $\Delta$ vs. $x / l$ for $p=2$ and $\mu=0$.

For $x \gtrsim l, G_{b}(x, 0)$ is exponentially suppressed. These results indicate an asymptotic scaling behavior given by

$$
G_{b}(x, 0) \approx l^{-1 / 2} g(x / l) .
$$

The region where $G_{b}(x, 0)$ appears to rapidly vanish, i.e., for $x / l \approx 1$ in Fig. 15, corresponds to the region where $\mu_{\mathrm{eff}} \approx 1$, cf. Eq. (36), which is the value of the chemical potential corresponding to the superfluid to empty state transition, where the particle density of the ground state vanishes. We thus expect that, for generic values of $\mu$ and $p$, the region around $x=x_{c}=l(1-\mu)^{1 / p}$, where $\mu_{\text {eff }}\left(x_{c}\right)=1$, develops critical modes related to a low-density Mott transition. The effective chemical potential can be expanded around $x_{c}$ as

$$
\mu_{\mathrm{eff}}=\mu+(x / l)^{p}=1+(1-\mu) p \frac{x-x_{c}}{l}+O\left[\left(x-x_{c}\right)^{2}\right] .
$$

Thus, the behavior around $x_{c}$ is essentially analogous to that arising at the low-density Mott transition $\mu=1$ in the presence of a linear potential $V_{l} \sim r / l$. Around $x_{c}$, critical modes should appear with length scale $\xi \sim l^{\sigma}$, where $\sigma$ is the exponent associated with a linear external potential. The value of $\sigma$ can be inferred by RG arguments analogous to those leading the determination of the trap exponent $\theta$ at the low-density Mott transition [23], which give $\sigma=1 / 3 .^{2}$ We thus expect that the transition region around $x=x_{c}$ enlarges as

$$
\Delta x \sim l^{1 / 3},
$$

\footnotetext{
2 The exponent $\sigma$ can be determined by a RG analysis of the perturbation corresponding to a linear potential $V_{l}(x)=u x$, i.e., $\int d^{d} x d t V_{l}(x)|\phi(x)|^{2}$, at the fixed point of the continuous theory describing the Mott transition [11]. The exponent $\sigma$ is related to the RG dimension $y_{u}$ of the parameter $u$, which can be obtained from the relations $y_{u}-1=d+z-y_{|\phi|^{2}}=y_{\mu}=2$, thus $y_{u}=3$, and therefore $\sigma \equiv 1 / y_{u}=1 / 3$ for $d=1$ and $d=2$.
} 


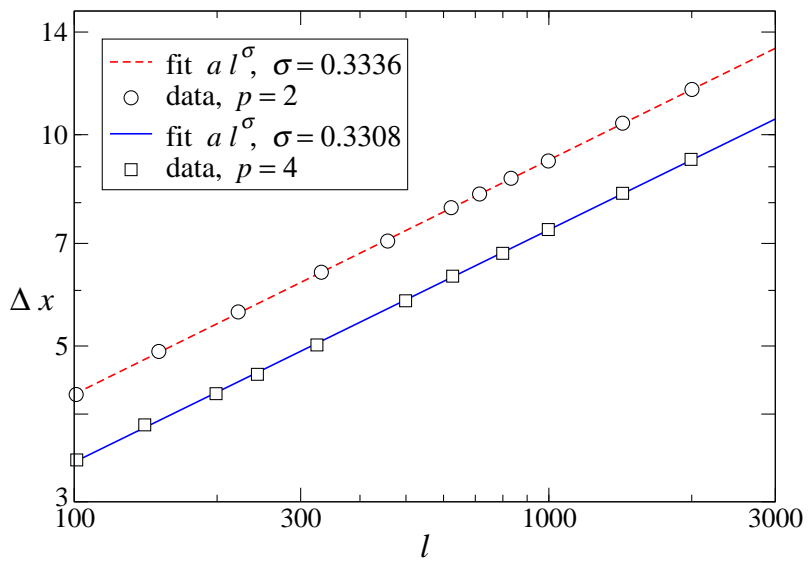

FIG. 16: $\Delta x$, defined by Eq. (66), vs. $l$ for $p=2,4$ and $\mu=0$. The full lines shows a fit to $\Delta x=a l^{\sigma}$ leaving free the exponent $\sigma$. The resulting estimates of $\sigma$ are in perfect agreement with the RG prediction $\sigma=1 / 3$ independently of $p$.

independently of the power-law $p$ of the confining potential. We study this phenomenon numerically by computing $G_{b}(x, 0)$ for $l$ s corresponding to odd peaks of $\Delta$; we take

$$
\Delta x \equiv l-x_{\max },
$$

where $x_{\max }$ is the abscissa of the rightmost maximum of $x^{1 / 2} G_{b}(x, 0)$; the results agree very well with $\Delta x \sim l^{1 / 3}$, see Fig. 16.

\section{Quantum entanglement in the superfluid phase}

We divide the chain in two parts of length $l_{A}$ and $L-l_{A}$, and consider the von Neumann entanglement entropy $S\left(l_{A} ; L\right)$ for open boundary conditions. In the absence of the trap and for open boundary conditions, the von Neumann entanglement entropy is essentially determined by the conformal field theory which describes its continuum limit [32],

$$
S\left(l_{A} ; L\right) \approx \frac{c}{6} \ln \left[L \sin \left(\pi l_{A} / L\right)\right]+E(\mu)
$$

where $c=1$ is the central charge corresponding to a relativistic free boson field theory. The $O(1)$ term, $E(\mu)$, depends on $\mu$; it is exactly known, see Eq. (B11) (notably, $E(0)=0.287769699 \ldots)$. We consider specifically the halflattice von Neumann entanglement entropy,

$$
S(L / 2 ; L)=\frac{1}{6} \ln L+E(\mu)+O(1 / L) .
$$

As shown in App. B, the amplitude of the $O(1 / L)$ correction is generally modulated by a function of $\bar{\phi} \equiv$ $2\{[(L+1) f+1] / 2\}$ (where $\{x\} \equiv x-\lfloor x\rfloor$ is the fractional part of $x)$, giving rise to peculiar oscillations.
In the presence of a trapping potential, and for $L \rightarrow \infty$, our numerical results show the behavior

$$
\begin{aligned}
S_{1 / 2} & =\lim _{L \rightarrow \infty} S(L / 2 ; L) \\
& =\frac{1}{6} \ln \left[a_{e}(\mu) l\right]+E(\mu)+A_{o}(\mu, \bar{\phi}) / l+O\left(1 / l^{2}\right)
\end{aligned}
$$

for any $p$, where $E(\mu)$ is the same constant in the absence of the trap, cf. Eq. (68). Eq. (69) defines an entanglement length scale [23]

$$
\xi_{e} \equiv a_{e} l
$$

Some results for the amplitude $a_{e}$ are reported in Table I. For $p \rightarrow \infty$ we recover Eq. (68) with $L=2 l$, thus

$$
\lim _{p \rightarrow \infty} a_{e}(\mu)=2 .
$$

The amplitude $A_{o}$ of the $O(1 / l)$ term turns out to be a periodic function of the trap size, through the dependence on $\bar{\phi}$, i.e., the phase-like variable $\bar{\phi}$ defined in Eq. (58). An analogous behavior is found in the limit $p \rightarrow \infty$, i.e., in the case of a homogeneous system of size $L$ with open boundary conditions, see App. B.

We also mention that similar subleading oscillations are observed in the half-lattice entanglement entropy of the XX model with gradients [33], i.e., in the presence of a linear external field.

\section{E. Some notable relations}

We have already shown that in the gapless superfluid phase the asymptotic modulated power-law behavior of the gap and the particle density in the middle of the trap is largely universal, being independent of $\mu$ and $p$, apart from trivial normalizations. In the following we show that also the amplitudes for different values of $p$ are strictly related; they can be derived from the $p=\infty$ limit which corresponds to the homogeneous system with open boundary conditions, see App. B.

We note that, using the entanglement definition (70) of length scale, for any $|\mu|<1$ and any $p$ including $p \rightarrow$ $\infty$, the asymptotic behavior of the gap and the particle density can be written as

$$
\begin{aligned}
& \Delta \approx \frac{\pi \sqrt{1-\mu^{2}}}{\xi_{e}} t(\phi), \\
& \left\langle n_{0}\right\rangle-f \approx \frac{1-\bar{\phi}}{\xi_{e}},
\end{aligned}
$$

with $\phi$ and $\bar{\phi}$ defined in Eqs. (47) and (58) respectively. Indeed, one can check that, within the high accuracy of our numerical estimates, the results reported in Table I satisfy the relations

$$
\begin{aligned}
& a(\mu ; p)=\frac{\pi \sqrt{1-\mu^{2}}}{a_{e}(\mu ; p)}, \\
& b(\mu ; p)=\frac{1}{a_{e}(\mu ; p)} .
\end{aligned}
$$


Moreover, Eqs. (72) and (73) reproduce the results for the homogeneous system with open boundary conditions, see App. B, by replacing the entanglement length scale $\xi_{e}$ with $L+1$, with $\phi$ and $\bar{\phi}$ given by the corresponding expressions (B2) and (B5) respectively.

These results provide a strong numerical evidence of the following statement: in the superfluid phase the asymptotic trap-size dependence of smooth observables can be obtained by replacing $L$ (or, more precisely $L+1$ ) with $\xi_{e}$ in the asymptotic behavior of the homogeneous system of size $L$ with open boundary conditions.

Another notable numerical relation is found at $\mu=0$ : the numerical data of the half-lattice entanglement $S_{1 / 2}$ in the presence of the trap indicates that the entanglement length scale $\xi_{e}$, cf. Eq. (70), is exactly given by

$$
\xi_{e}=2 Q_{l}^{*} l
$$

for any $p$, where $Q_{l}^{*}$ is the asymptotic periodicity of the level crossings, cf. Eq. (53), which is exactly derived from the LDA of the total particle number, i.e., from the relation

$$
1 / Q_{l}^{*}=\frac{2}{\pi} \int_{0}^{1} d x \arccos x^{p}=\frac{2 p \Gamma\left(\frac{1+p}{2 p}\right)}{\sqrt{\pi} \Gamma\left(\frac{1}{2 p}\right)} .
$$

We are quite confident that Eq. (76) holds, since the numbers reported in Table I show that it is verified within the numerical accuracy of our estimates of the amplitude $a_{e}$ for $p=2$ and $p=4$, which is $O\left(10^{-7}\right)$. Moreover, it correctly reproduces the $p \rightarrow \infty$ limit $\xi_{e}=2 l$.

In addition, we find that at $\mu=0$ the behavior of the half-lattice entanglement $S_{1 / 2}$ in the presence of the trap turns out to be consistent with the following formula

$$
S_{1 / 2}=\frac{1}{6} \ln \xi_{e}+E(0)+\frac{\pi}{4 \xi_{e}} A_{o}(\bar{\phi})+O\left(1 / \xi_{e}^{2}\right)
$$

for any $p$, where

$$
A_{o}(\mu ; \bar{\phi})=\left\{\begin{array}{cl}
1+c(\bar{\phi}-1 / 2) & \text { for } \quad 0<\bar{\phi}<1 \\
-1-c(3 / 2-\bar{\phi}) & \text { for } \quad 1<\bar{\phi}<2
\end{array}\right.
$$

with $c \approx-0.150$ for $p=2$ and $c \approx-0.044$ for $p=4$. Note that the behavior of the homogeneous system with open boundary condition, cf. Eq. (B15), is obtained by replacing $\xi_{e} \rightarrow L+1$ for the values $\bar{\phi}=1 / 2,3 / 2$, which are the only possible values for the homogeneous system in a chain with even $L$, corresponding to odd and even $L / 2$ respectively.

\section{F. Discussion}

The trap-size dependence of the half-lattice entanglement shows that the confining potential induces a length scale which behaves as $\xi \sim l$ for any power of the potential, at least for smooth observables. In the framework of the TSS, this would imply that the trap exponent is $\theta=1$ independently of $p$. This value of $\theta$ is also obtained from the trap-size dependence of the gap, which scales as $\Delta \sim l^{-1}$, apart from a periodic dependence on $l$ of its amplitude, cf. Eq. (56). Indeed, the exponent of the power behavior is expected to be $z \theta$, and in the superfluid region we have $z=1$. A consistent scaling is also observed in the case of the one-particle density matrix $G_{b}\left(x_{i}, x_{j}\right)$, see Sec. IV C 5. On the other hand, the density correlations show clearly a coupling with modes at the Fermi momentum $k_{F}=\pi f$, which are apparently characterized by a different length scale $\xi_{\mathrm{f}}$, scaling as $\xi_{\mathrm{f}} \sim l^{\zeta}$ with $\zeta=p /(p+1)$.

These results may be explained by the nontrivial coupling of the confining potential with the free bosonic field $\phi(x)$ of the continuum theory, due to the fact that in the continuum limit the spin operator $\sigma_{i}^{z}$ can be written as a sum of a slow contribution proportional to $\partial_{x} \phi$ and a rapidly oscillating contribution proportional to $e^{2 i k_{F} x} \phi$, see, e.g., Refs. [25, 26]. Assuming that the latter term is suppressed for smooth or global quantities, such as the half-lattice entanglement entropy and the gap, where its effects should get averaged out, we may argue that $\theta=1$ is indeed the expected trap exponent. A heuristic argument may be obtained by noting that the perturbation $\int d x V(x)(d \phi(x) / d x)$ can be rewritten as $\int d x(d V(x) / d x) \phi(x)$ by integration by parts, whose first-order perturbation vanishes because $V(x)$ is even in $x$. We then expect that the leading contribution comes from next-to-leading terms, like $\int d x V(x)\left(d^{2} \phi(x) / d x^{2}\right)$. RG arguments applied to this perturbation, taking into account that $\phi$ is a free Bose field, lead to $\theta=1$ independently of $p$, which is the result emerging from the numerical diagonalization of the Hamiltonian. On the other hand, correlators which are nontrivially coupled to the modes at $k_{F}$ may show a different length scale due to the coupling of the confining potential with the staggered term in $\sigma^{z}$. This is indeed what we observe in the correlation of density operators which are directly related to the operator $\sigma^{z}$.

\section{MODULATED TSS AT THE $n=1$ MOTT INSULATOR TO SUPERFLUID TRANSITION}

In the section we study the effects of the trap at the $n=1$ Mott insulator to superfluid transition, i.e., at $\mu_{c}=-1$, where the filling factor of the homogeneous system is $f=1$. The confining potential $V(x)$ gives rise to a change of the particle density from $\langle n\rangle \approx 1$ in the middle of the trap to $\langle n\rangle=0$ at large distance, passing through the gapless superfluid phase, see Fig. 9. Specifically, for $\mu=-1$, the particle density appears to vanish when $\mu_{\text {eff }} \gtrsim 1$, cf. Eq. (36), thus, $x / l \gtrsim 2^{1 / p}$.

We recall that the behavior around $\mu=-1$ of the homogeneous $\mathrm{BH}$ model without trap is essentially analogous to that at $\mu=1$, because of the invariance under the particle-hole exchange. At the $n=1$ Mott insulator 


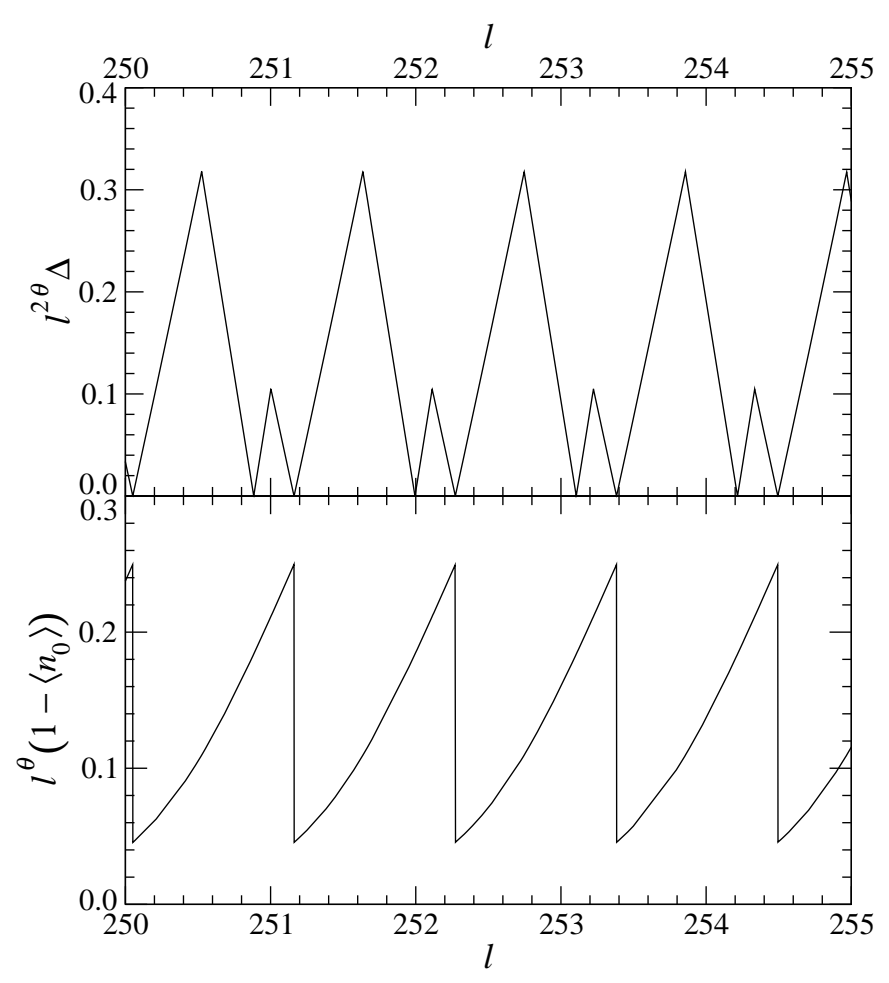

FIG. 17: The rescaled energy gap $l^{2 \theta} \Delta$ (above) and the rescaled particle density in the middle of the $\operatorname{trap} l^{\theta}\left(1-\left\langle n_{0}\right\rangle\right)$ (below) vs. $l$ for $\mu=-1$ and $p=2$, whose trap exponent is $\theta=1 / 2$. Results for $p=4$ and $p=6$ are similar.

to superfluid quantum transition, the critical exponents $z$ and $y_{\mu}$ and the trap-size exponent $\theta$ are the same as those at $\mu=1$, i.e., $z=2, y_{\mu}=2$ and $\theta=p /(2+p)$. However, the particle-hole symmetry does not hold in the presence of the trapping potential, and the asymptotic trap-size dependence appears more complicated at the $n=1$ Mott transition. This is essentially related to the presence of level crossings at finite values of the trap size, where the gap vanishes, as already found in the superfluid region, for $|\mu|<1$. As we shall see, the resulting trap-size dependence can be cast in the form of a modulated TSS, that is a TSS controlled by the same exponents as those at the low-density Mott transition, but modulated by periodic functions of the trap size.

\section{A. Modulated TSS of the gap}

Results for the gap and the particle density at the middle of the trap are shown in Fig. 17. They suggest periodic asymptotic behaviors of the scaling quantities $l^{2 \theta} \Delta$ and $l^{\theta}\left(1-\left\langle n_{0}\right\rangle\right)$ as functions of $l$, with a period given by the interval between two even (or odd) zeroes of the gap, and a marked difference between even- and oddnumbered crossings and peaks.

In the large- $l$ limit we find that the interval between

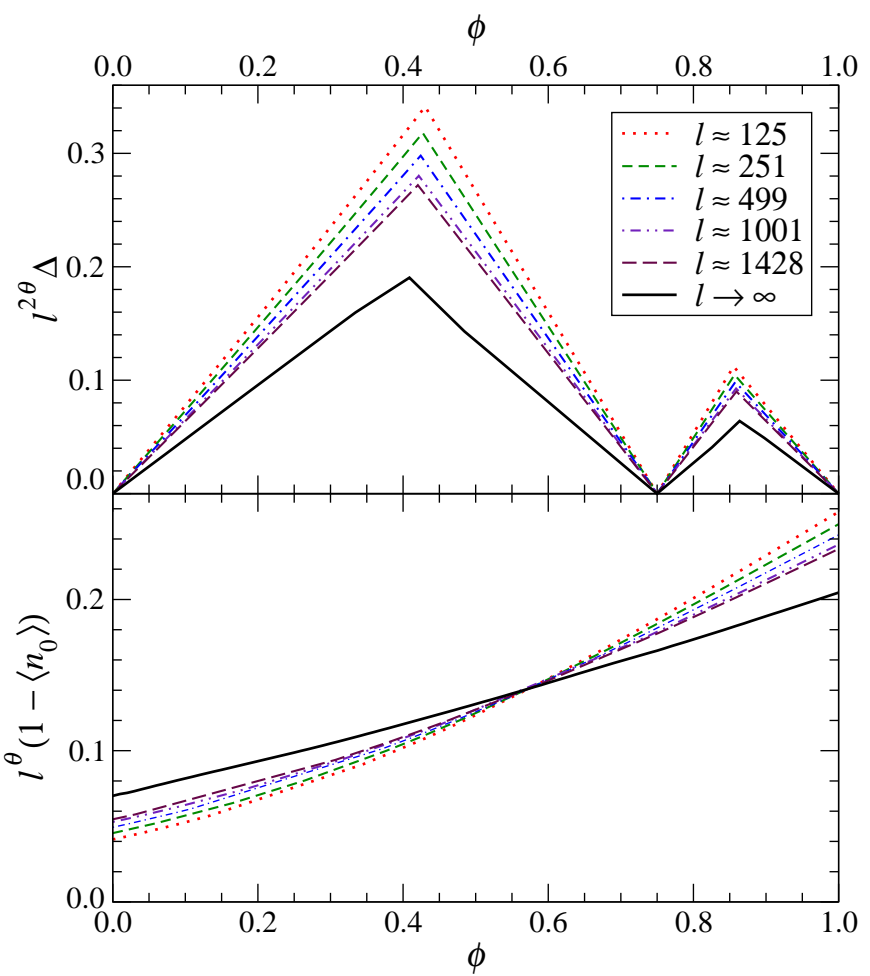

FIG. 18: $l^{2 \theta} \Delta$ (above) and $l^{\theta}\left(1-\left\langle n_{0}\right\rangle\right)$ (below) vs. $\phi$, defined in Eq. (82), for $p=2(\theta=1 / 2)$. The extrapolation to $l \rightarrow \infty$ is obtained by assuming $O\left(l^{-\theta / 2}\right)$ leading corrections. In the legends $l$ is rounded to the nearest integer for the sake of presentation.

two even zeroes approaches a constant value, i.e.,

$$
P_{l}^{(k)} \equiv l_{0}^{(2 k+2)}-l_{0}^{(2 k)}=P_{l}^{*}+O\left(l^{-2}\right) .
$$

The large- $l$ limit is estimated to be

$$
\begin{aligned}
& P_{l}^{*} \cong 1.11072073 \text { for } p=2, \\
& P_{l}^{*} \cong 1.10243940 \text { for } p=4 \\
& P_{l}^{*} \cong 1.08184087 \text { for } p=6 .
\end{aligned}
$$

Analogously to the trap-size dependence in the superfluid region, see Sec. IV D, the asymptotic interval $P_{l}^{*}$ can be estimated using the LDA of the total particle number, cf. Eq. (40). We find again identical results, showing that the LDA of the total particle density is asymptotically exact in the large- $l$ limit also at the $n=1$ Mott transition. The $p \rightarrow \infty$ limit of $P_{l}^{*}$ can be easily computed using the LDA, obtaining $P_{l}^{*} \rightarrow 1$ for $p \rightarrow \infty$. Corrections to the LDA of the particle density are discussed below.

The asymptotic periodic properties clearly emerge from the results shown in Figs. 18, 19 and 20, where $l^{2 \theta} \Delta$ and $l^{\theta}\left(1-\left\langle n_{0}\right\rangle\right)$, for $p=2,4,6$ respectively, are plotted versus the phase-like variable

$$
\phi=\frac{l-l_{0}^{(2 k)}}{l_{0}^{(2 k+2)}-l_{0}^{(2 k)}}, \quad l_{0}^{(2 k)} \leq l<l_{0}^{(2 k+2)},
$$




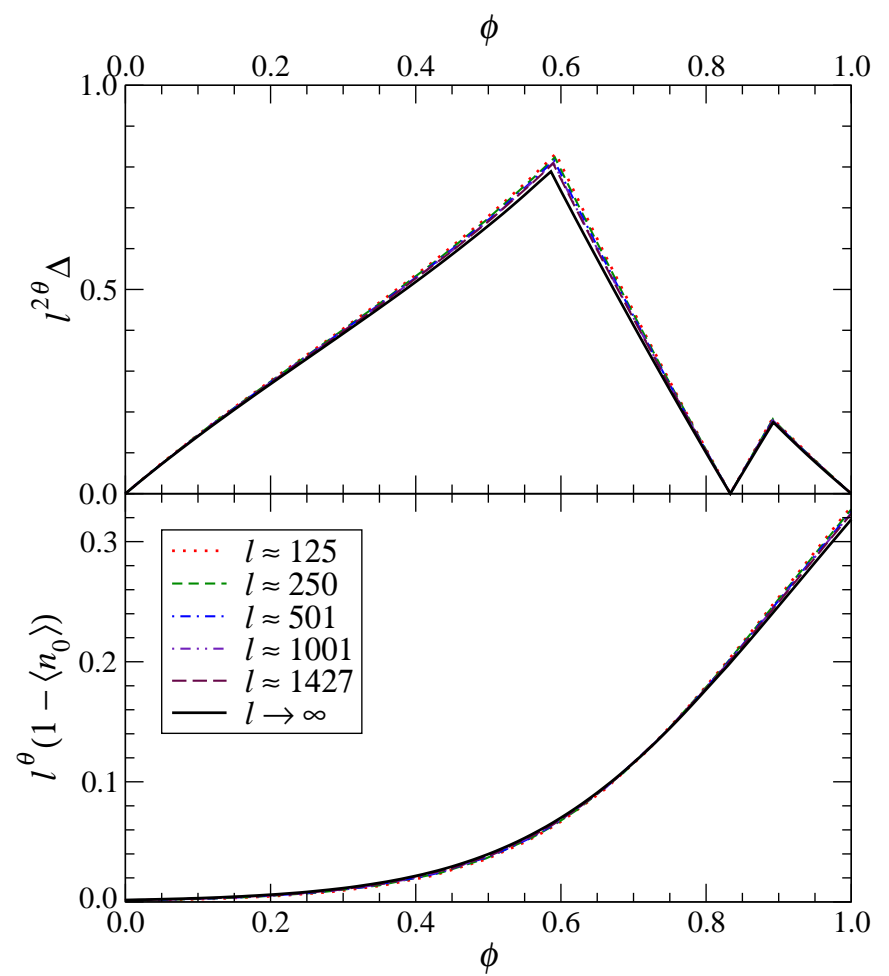

FIG. 19: $l^{2 \theta} \Delta$ (above) and $l^{\theta}\left(1-\left\langle n_{0}\right\rangle\right)$ (below) vs. $\phi$, defined in Eq. (82), for $p=4(\theta=2 / 3)$.

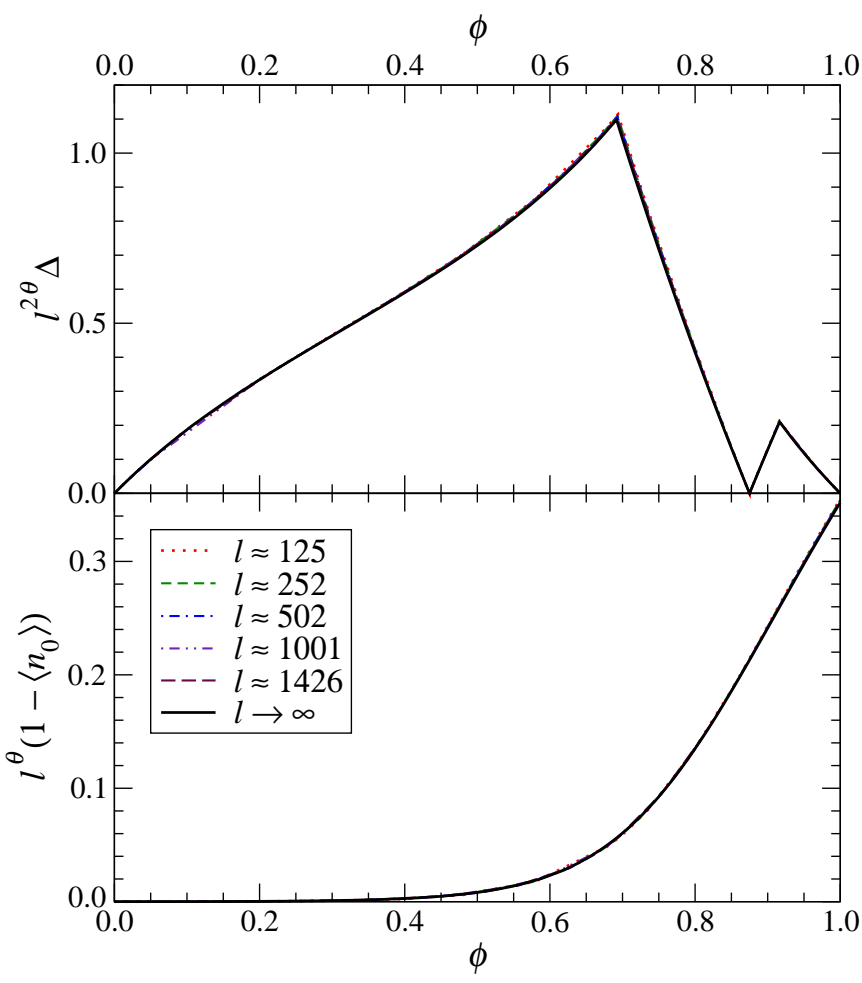

FIG. 20: $l^{2 \theta} \Delta$ (above) and $l^{\theta}\left(1-\left\langle n_{0}\right\rangle\right)$ (below) vs. $\phi$, defined in Eq. (82), for $p=6(\theta=3 / 4)$. thus $0 \leq \phi<1$.

The results for the gap show that the quantity $l^{2 \theta} \Delta$ approaches an asymptotic periodic function $A_{\Delta}(\phi)$ in the large- $l$ limit. Therefore, they provide a clear evidence for a modulated asymptotic behavior

$$
\Delta \approx A_{\Delta}(\phi) l^{-2 \theta}\left[1+O\left(l^{-\kappa}\right)\right]
$$

with $\kappa \approx \theta / 2$ (see below). Note that, for $p=2$, the scaling of the gap is controlled by the same exponent found in the region $|\mu|<1$, because we have $2 \theta=1$; on the other hand, for $p=4$ and $p=6$ we have respectively $2 \theta=4 / 3$ and $2 \theta=3 / 2$, which are easily differentiated from 1 .

We should note that in these calculations the trap of size $l$ is centered in the middle of the chain of size $L$; more precisely we consider odd $L \mathrm{~s}$ to have the center of the trap coincide with the middle site of the chain. Even $L \mathrm{~s}$ would instead correspond to traps centered between the two central sites. Unlike the previous cases, at $\mu=-1$ this difference must be taken into account, but it gives only rise to an interchange of the role of the even and odd zeroes of the gap. Therefore, in the case of even $L \mathrm{~s}$, one may simply redefine the phase-like variable as

$$
\phi=\frac{l-l_{0}^{(2 k-1)}}{l_{0}^{(2 k+1)}-l_{0}^{(2 k-1)}}, \quad l_{0}^{(2 k-1)} \leq l<l_{0}^{(2 k+1)} .
$$

The two different definitions (82) and (84) remove the dependence on the parity of $L$ (cf. App. A) from the modulation functions $A(\phi)$ of the asymptotic behaviors of the observables, such as $A_{\Delta}(\phi)$. Using, e.g., definition (82) for all $L$ s would lead to $\left.A(\phi)\right|_{\text {even } L}=\left.A\left(\left\{\phi+\phi_{0}\right\}\right)\right|_{\text {odd } L}$, where $\{x\} \equiv x-\lfloor x\rfloor$ is the fractional part of $x$. Note that centering the trap at an arbitrary distance $q$ from the nearest site of the chain would modify significantly the modulation.

By definition, $A_{\Delta}(0)=A_{\Delta}(1)=0 . A_{\Delta}$ has another zero $\phi_{0}$ corresponding to the odd level crossings. Computations of $\phi_{0}$ for the XX model and $p=2,4,6$ are shown in Fig. 21, where they are plotted vs $L^{-\theta}$. They asymptotically approaches the value $\phi_{0} \cong 0.750000$ for $p=2, \phi_{0} \cong 0.833333$ for $p=4, \phi_{0} \cong 0.875000$ for $p=6$, and $\phi_{0} \cong 0.916667$ for $p=10$, with $O\left(l^{-2 \theta}\right)$ corrections. Note that these values are compatible with the simple formula

$$
\phi_{0}=(p+1) /(p+2) .
$$

Other features of the modulation function $A_{\Delta}(\phi)$, such as the location of the large and small peaks, $\phi_{l}$ and $\phi_{s}$ respectively, and its peak values $A_{\Delta}\left(\phi_{l, s}\right)$, are approached with power-law scaling corrections $O\left(l^{-\kappa}\right)$. We find $\kappa \approx 1 / 4$ for $p=2, \kappa \approx 1 / 3$ for $p=4$, and $\kappa \approx 3 / 8$ for $p=6$, which are consistent with $\kappa=\theta / 2$. See, e.g., Figs. 22 and 23. Note that $\kappa=\theta / 2$ should be considered as a phenomenological result, because we do not have theoretical arguments to derive it.

A faster approach to scaling is found for the ratio $\Delta_{l} / \Delta_{s}$ between subsequent large and small peaks of the 

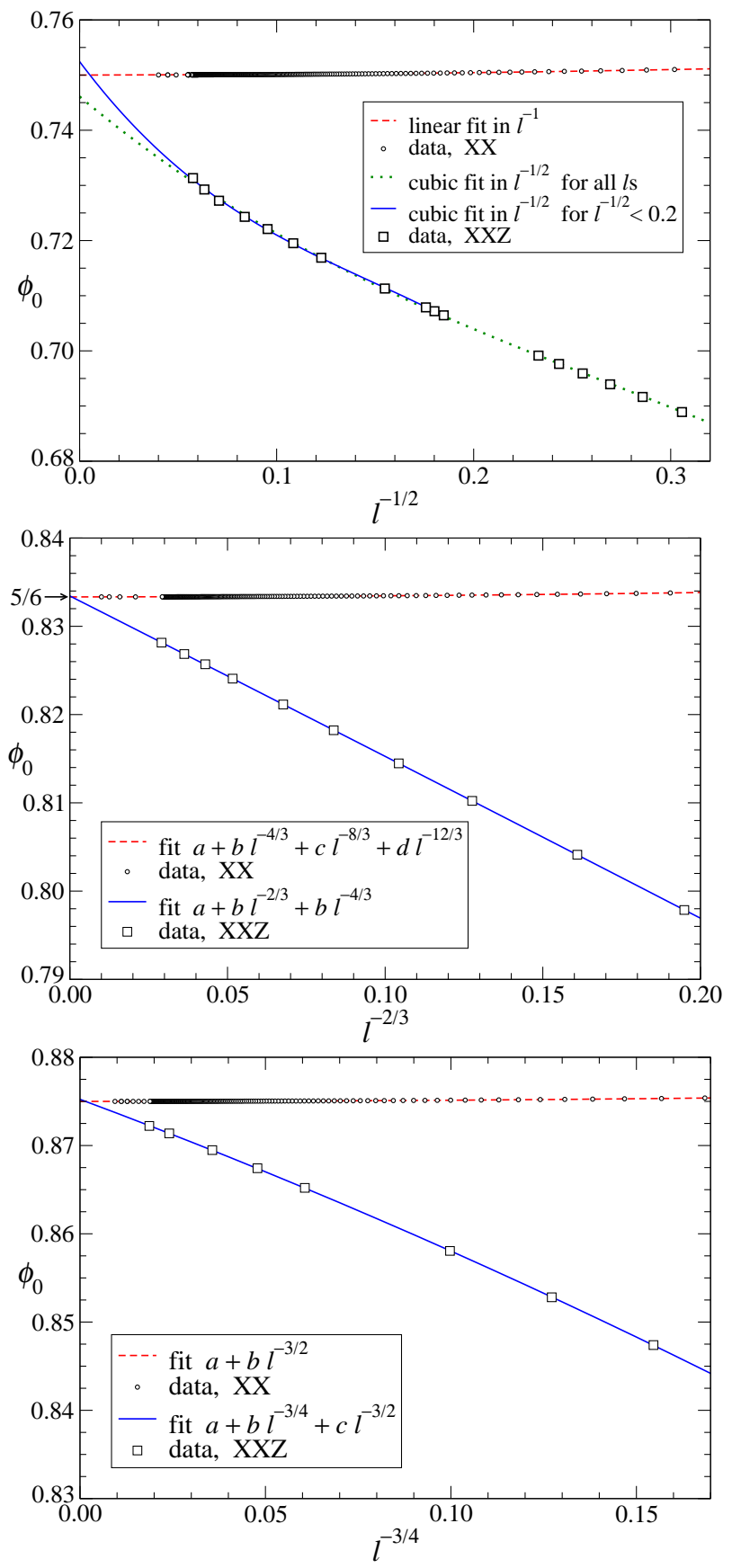

FIG. 21: $\phi_{0}$ vs. $l^{-\theta}$, for the XX model (6) at $\mu=-1$ and the XXZ model (33) at $j_{z}=-1 / 2$ and $\mu=-1 / 2$, for $p=2$ (above), $p=4$ (middle), and $p=6$ (below).

gap, which is expected to be universal (essentially because it is independent of normalizations), see Fig. 24. (We compute the ratio at $l \mathrm{~s}$ corresponding to $\Delta_{s}$, obtaining $\Delta_{l}$ by cubic spline interpolation.) We find a behavior compatible with

$$
\Delta_{l} / \Delta_{s}=a+b l^{-\theta}+O\left(l^{-2 \theta}\right)
$$

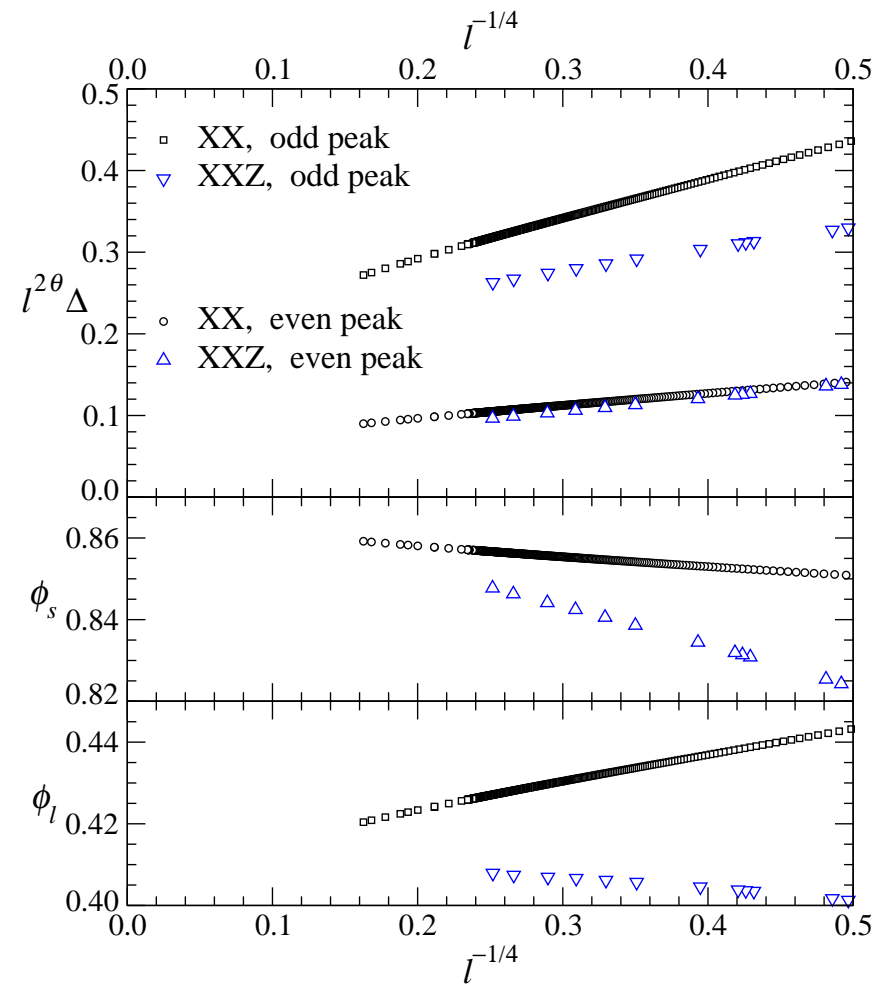

FIG. 22: Scaling of the large (odd) and small (even) peaks of the gap and their location $\phi_{l}$ and $\phi_{s}$ for $p=2$, for the XX model (6) and the XXZ model (33) at $j_{z}=-1 / 2$.

It is worth noting that, in the case $p=2, A_{\Delta}(\phi)$ has apparently a simple form: it is approximately formed by two reflected similar triangles, as shown in Fig. 18. Indeed, this shape would require $\Delta_{l} / \Delta_{s}=\phi_{0} /\left(1-\phi_{0}\right)=$ 3 , while we obtain a very close value $\Delta_{l} / \Delta_{s}=3.013$ from the $l \rightarrow \infty$ extrapolation of the results at fixed $l$.

The behavior in the large- $p$ limit can be guessed from the results obtained at finite $p$ shown above. Note that they do not apparently approach the finite-size behavior of a homogeneous $\mathrm{BH}$ model on a chain of size $L=2 l$ with open boundary conditions, as one may naively expect. For example, the gap of the homogeneous $\mathrm{BH}$ model at $\mu=-1$ behaves as $\Delta=O\left(1 / L^{2}\right)$ without showing level crossings. ${ }^{3}$ As a consequence, the phase-like variable does not have any corresponding quantity in the BH model without trap. Thus the relations between the large- $p$ limit and the finite-size behavior of the homogeneous BH model are not straightforward.

Finally, let us mention that the gap at $\mu<-1$ shows nontrivial trap-size dependence as well, because the phenomenon of the level crossings persists. The numerical

\footnotetext{
3 This result can be easily derived using the particle-hole exchange symmetry, and the corresponding result at the low-density Mott transition, at $\mu=1$, see, e.g., Eq. (19).
} 


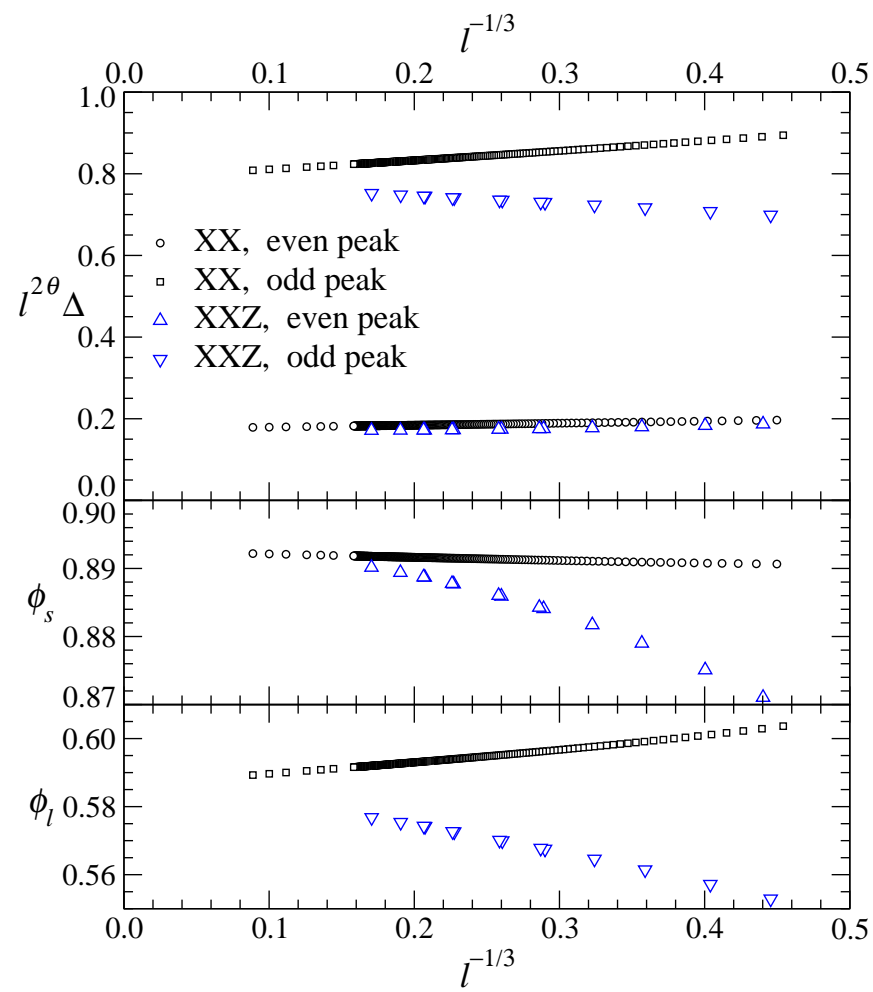

FIG. 23: Scaling of the large (odd) and small (even) peaks of the gap and their location $\phi_{l}$ and $\phi_{s}$ for $p=4$, for the XX model (6) and the XXZ model (33) at $j_{z}=-1 / 2$.

results at $\mu=-1.2$ show again a periodic structure of the gap, which gets suppressed as $1 / l$ for any $p$. They show large and small peaks, but the smallest one gets rapidly suppressed in the large- $l$ limit. We observe that the large peak turns out to scale as $\Delta_{l} \sim 1 / l$ for $p=2$ and $p=4$, while the small peak decreases as $\Delta_{s} \sim \exp (-a l)$; therefore, the ratio $\Delta_{s} / \Delta_{l}$ vanishes as $l \rightarrow \infty$; in the same limit, $\phi_{0} \rightarrow 1$. Although for $\mu<-1$ the homogeneous system without trap has a gap proportional to $\mu_{s} \equiv-\mu-1$, see, e.g., Ref. [25], here we find that the periodic trap-size dependence of the gap tends to be suppressed as $1 / l$ in the large- $l$ limit. This is related to the fact that, in the presence of the trap, including its large- $l$ limit, we have always some critical regions, for example the superfluid regions between the $\left\langle n_{x}\right\rangle=1$ and $\left\langle n_{x}\right\rangle=0$ plateaus, for

$$
(-1-\mu)^{1 / p} \lesssim|x| / l \lesssim(1-\mu)^{1 / p}
$$

which becomes larger and larger with increasing $l$. An analogous behavior is found for the trap-size dependence of the XY chain when the middle of the trap is in the quantum ferromagnetic phase [23].
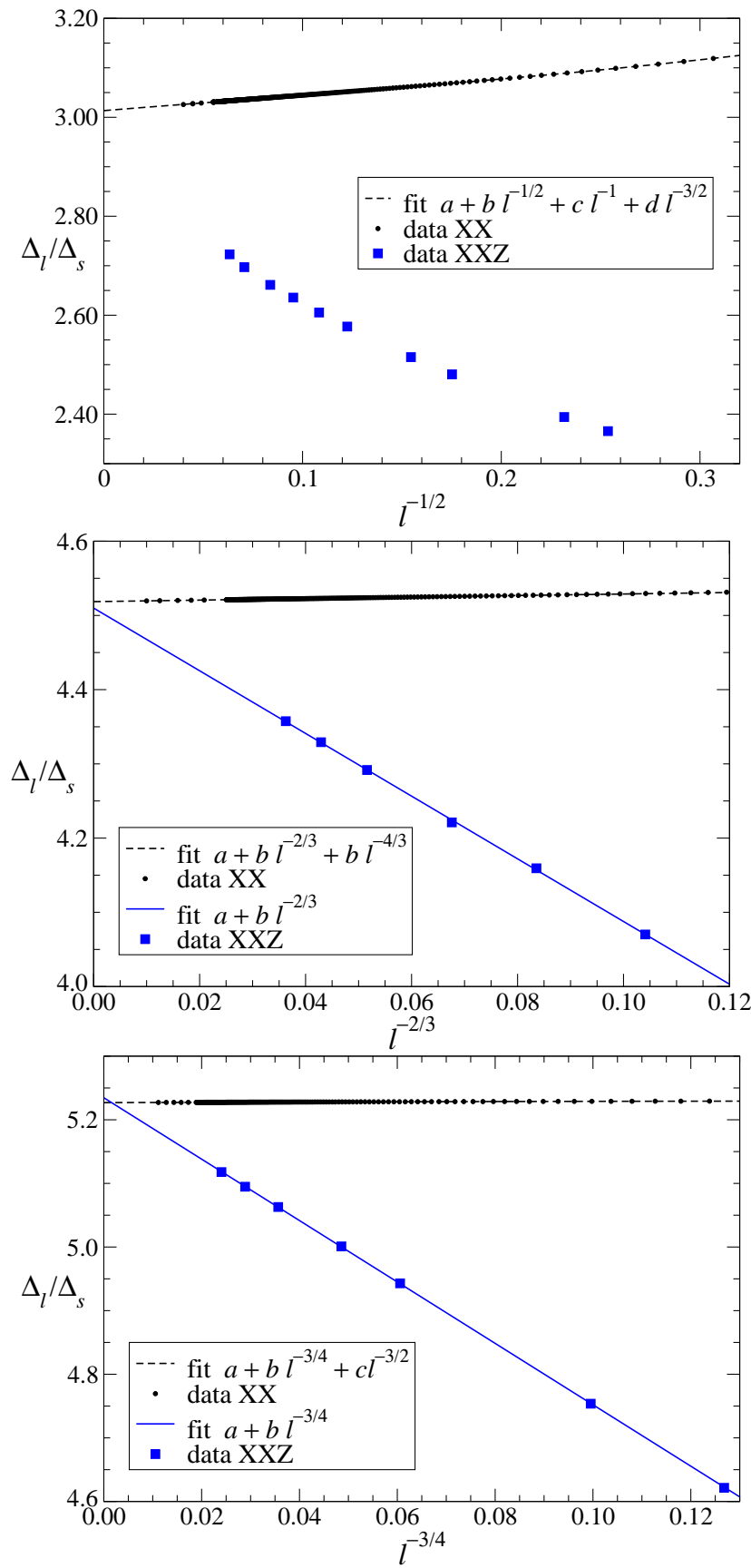

FIG. 24: $\Delta_{l} / \Delta_{s}$ vs. $l^{-\theta}$ for the XX model and for the XXZ model at $j_{z}=-1 / 2$, for $p=2$ (above), $p=4$ (middle) and $p=6$ (below).

\section{B. The particle density and its correlators}

Concerning the particle density, we recall that $\left\langle n_{x}\right\rangle=1$ in the absence of the trap and the RG dimension of the particle density is $y_{n}=1$ at the Mott transition. Thus, TSS predicts the scaling behavior $\left\langle n_{0}\right\rangle-1 \sim l^{-\theta}$ for the particle density at the middle of the trap and $\mu=-1$. Like the gap, we find that the trap-size dependence of 

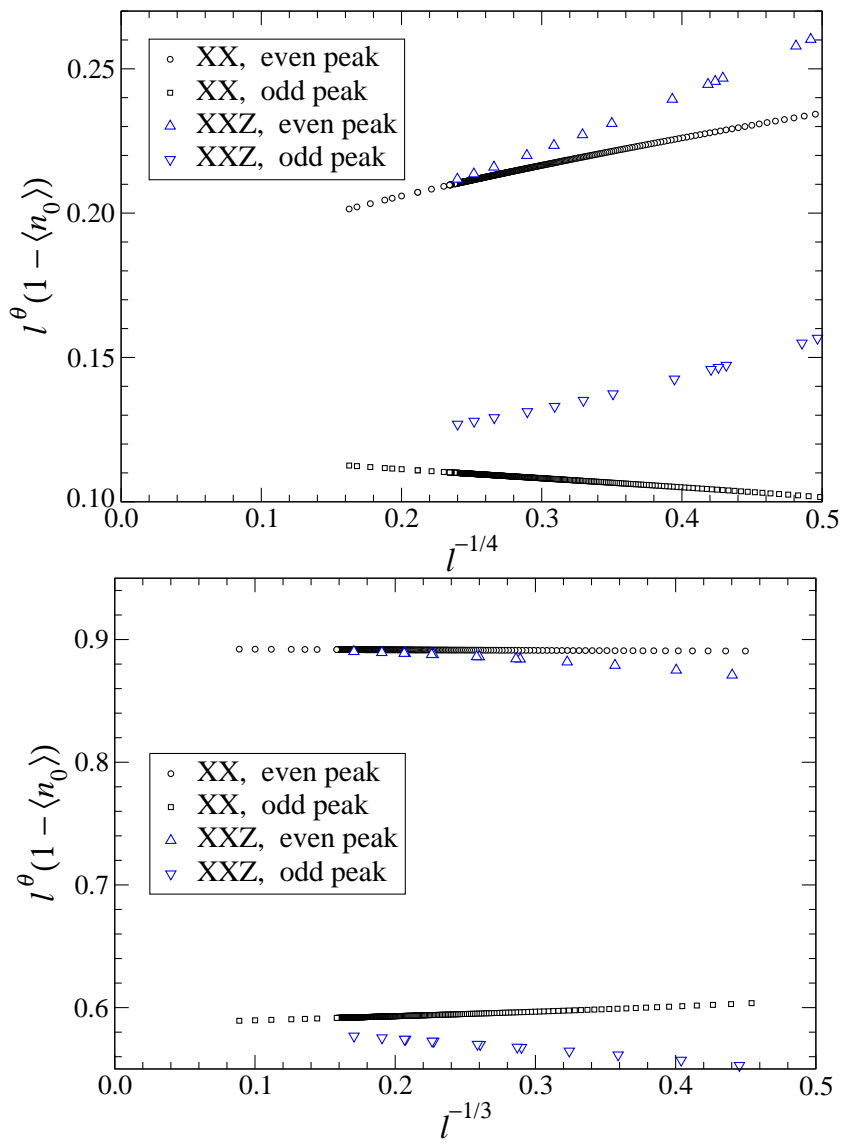

FIG. 25: Scaling of the particle density at the peaks of the gap for $p=2$ (above) and $p=4$ (below), for the XX model and the XXZ model (33) at $j_{z}=-1 / 2$.

$\left\langle n_{0}\right\rangle$ is described by a modulated TSS, i.e.,

$$
\left\langle n_{0}\right\rangle-1 \approx l^{-\theta} \mathcal{D}_{0}(\phi)
$$

where $\phi$ is the same phase-like variable defined for the scaling of the gap, cf. Eq. (82). Figs. 18, 19 and 20 show $l^{\theta}\left(1-\left\langle n_{0}\right\rangle\right)$ vs. $\phi$ for $p=2,4,6$ respectively. The approach to the asymptotic behavior is apparently characterized by power-law $O\left(l^{-\kappa}\right)$ corrections with $\kappa \approx \theta / 2$, analogously to the behavior of the gap. Notice that, unlike the superfluid case, the leading term has a nonzero average over the period. Results for the particle density at the peaks of the gap are shown in Figs. 25 for $p=2$ and $p=4$.

The spatial dependence of the particle density at large trap size turns out to be described by the following scaling behavior

$$
\left\langle n_{x}\right\rangle \approx \rho_{\mathrm{lda}}(x / l)+l^{-\theta} \mathcal{D}(X, \phi), \quad X=l^{-\theta} x .
$$

As already found in the superfluid region, the term depending on $x / l$ is given by the LDA of the particle density, cf. Eq. (37). It plays the role of an analytical contribution which must be subtracted to observe scaling in
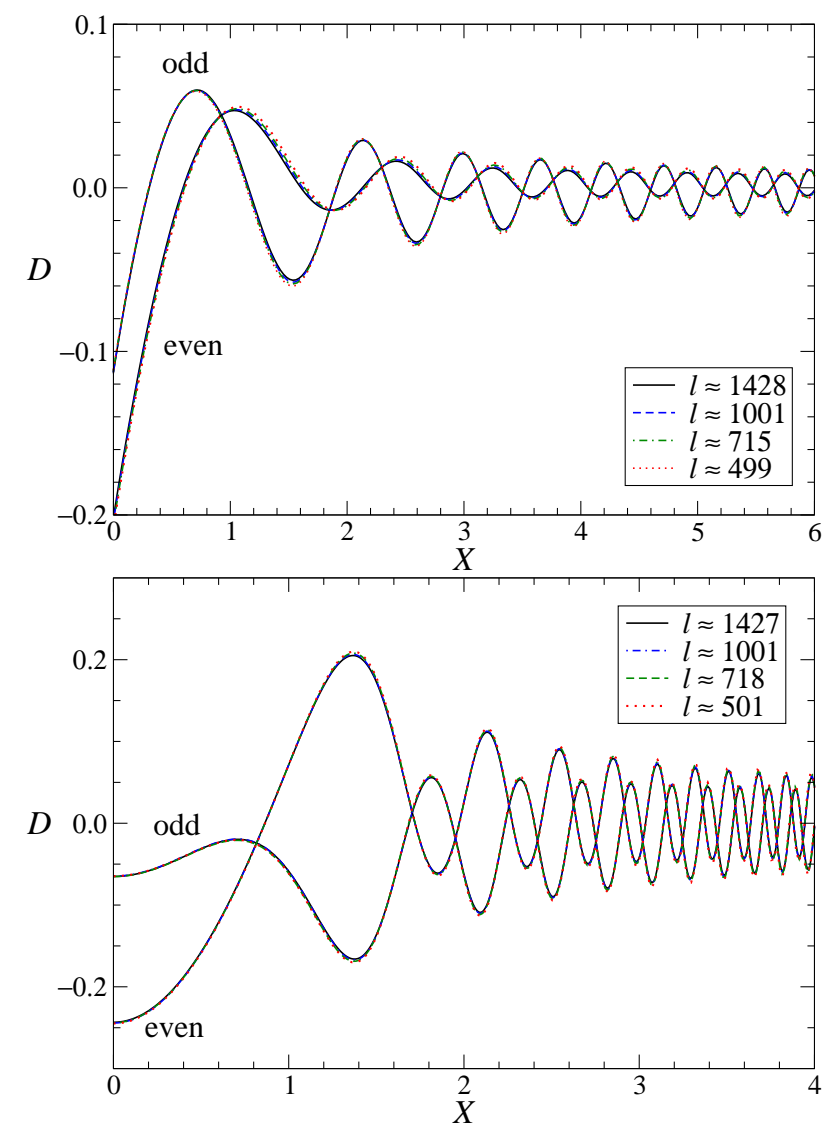

FIG. 26: The function $\mathcal{D}$ vs. $X=l^{-\theta} x$ at odd and even peaks of $\Delta$ at $\mu=-1$, for $p=2$ (above) and $p=4$ (below).

the expectation value of the density operators at phase transitions [23, 24]. Some results for the scaling function $\mathcal{D}(X, \phi)$ at the peaks of the gap are shown in Fig. 26 .

As already shown by the data at $\mu=-1$ reported in Fig. 9, the particle density is quantitatively dominated by its LDA at large trap size, which scales as $x / l$.

Assuming a modulated TSS, we expect that the density correlation behaves as

$$
G_{n}(x) \equiv\left\langle n_{0} n_{x}\right\rangle_{c}=l^{-2 \theta} \mathcal{G}_{n}(X, \phi) .
$$

This is confirmed by the numerical results. For example, Fig. 27 shows $l^{2 \theta} G_{n}(x)$ vs. $X$ for $p=2$ and several values of $l$ corresponding to peaks of $\Delta$ (i.e., $\phi=\phi_{l}$ or $\phi_{s}$ asymptotically). The approach to scaling is clearly observed.

\section{The one-particle density matrix}

The modulated TSS also applies to the critical behavior of the one-particle density matrix. We recall that the RG dimension of the bosonic field $b_{i}$ is $y_{b}=1 / 2$ at the Mott transitions. Consistently, the numerical results 


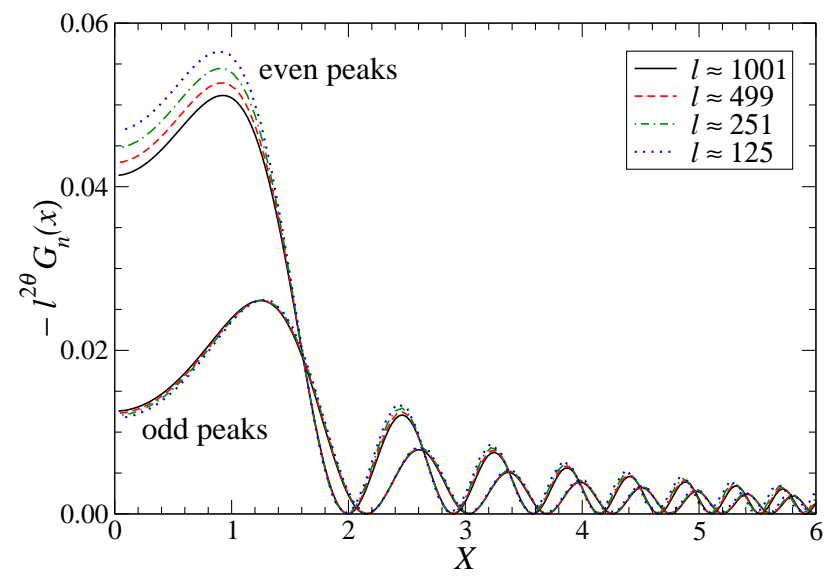

FIG. 27: The rescaled connected correlation $l^{2 \theta} G_{n}(x)$ vs. $X \equiv$ $x l^{-\theta}$ for $p=2, \mu=-1$, and $l$ s corresponding to even and odd peaks of $\Delta$. The data at different trap sizes clearly approach a TSS function when increasing $l$.
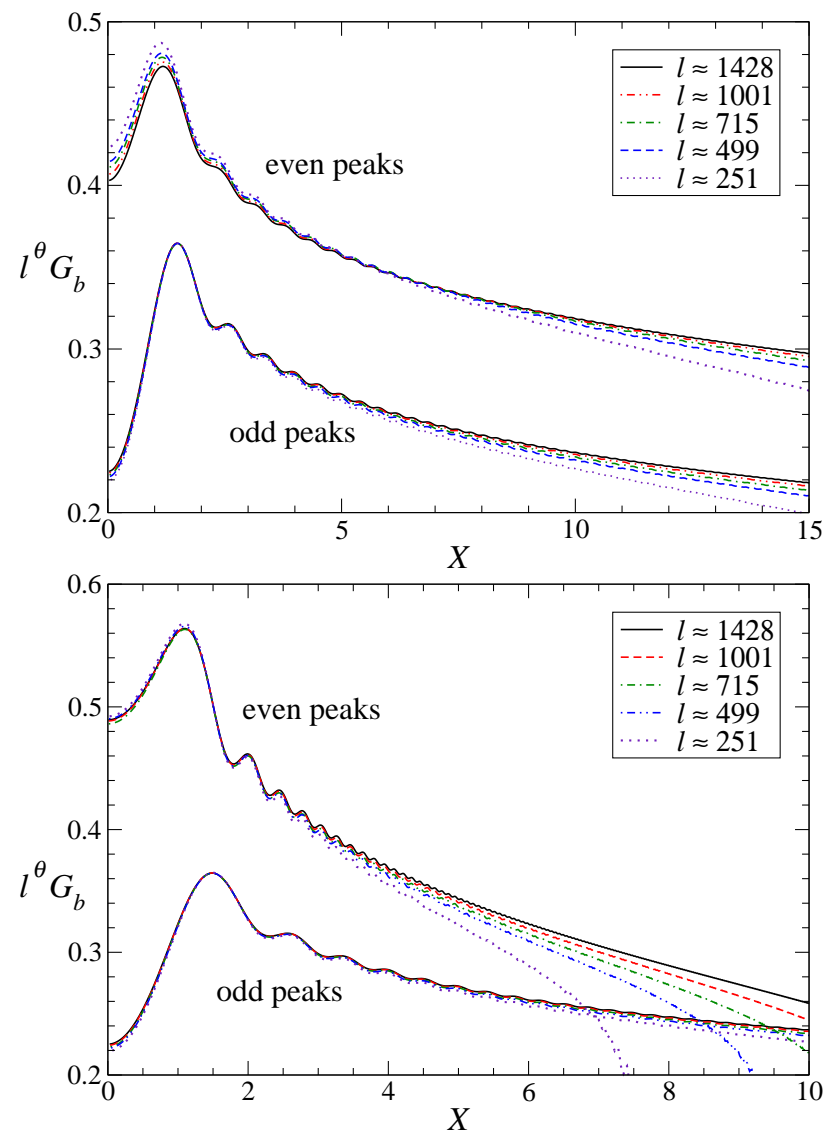

FIG. 28: The rescaled one-particle density matrix $l^{\theta} G_{b}(x)$ vs. $X \equiv x l^{-\theta}$ for $p=2$ (above) and $p=4$ (below), at $\mu=-1$, and for $l$ s corresponding to odd and even peaks of $\Delta$.

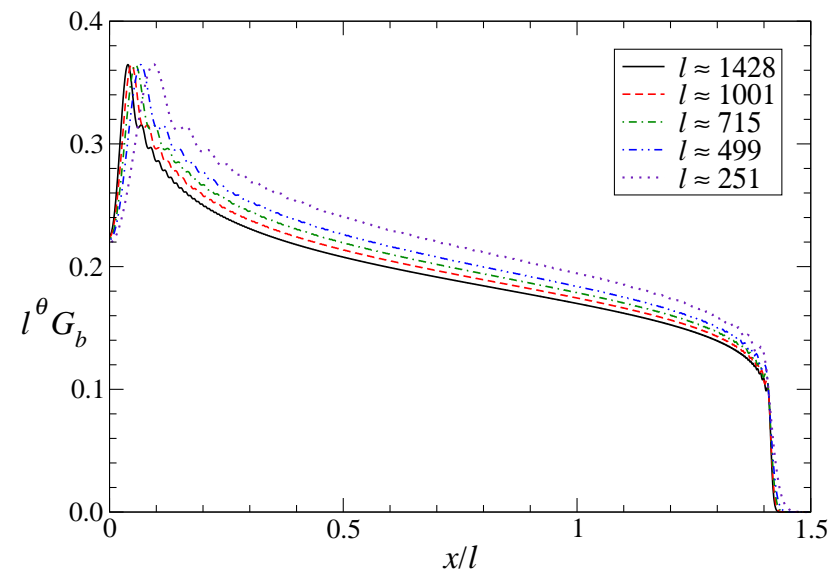

FIG. 29: $l^{1 / 2} G_{b}(x, 0)$ vs. $x / l$ for $p=2$ and $\mu=-1$. Data taken at the odd peaks of the gap.

show the behavior

$$
G_{b}(x, 0) \equiv\left\langle b_{0}^{\dagger} b_{x}\right\rangle=\left\langle\sigma_{0}^{-} \sigma_{x}^{+}\right\rangle \approx l^{-\theta} \mathcal{M}(X, \phi) .
$$

In Figs. 28 we plot $l^{\theta} G_{b}(x, 0)$ vs. $X$ for $p=2,4, \mu=-1$, and several values of $l$ corresponding to peaks of $\Delta$ (i.e., $\phi=\phi_{l}$ or $\phi=\phi_{s}$ asymptotically).

More generally, we may extend the modulated TSS including the dependence on $\hat{\mu} \equiv \mu+1$ around $\hat{\mu}=0$. Setting $\mu_{s}=l^{2 \theta} \hat{\mu}$, we expect

$$
G_{b}(x, 0) \approx l^{-\theta} \mathcal{M}\left(\mu_{s}, X, \phi\right) .
$$

Note that the modulated TSS is observed at fixed $X=x / l^{\theta}$, thus, since $\theta<1$, the region of $x \approx l$ gets hidden in the limit $X \rightarrow \infty$. Fig. 29 shows numerical results for $p=2$ versus $x / l$, where we clearly observe that the behavior for $x \gtrsim l$ is approximately scaling as $x / l$. This is essentially related to the simple scaling of the effective chemical potential, cf. Eq. (36). However, a more careful analysis shows another interesting scaling behavior around the region

$$
x \approx x_{c} \equiv 2^{1 / p} l,
$$

where the spatial dependence of the effective chemical gives rise to a transition from the superfluid phase to the $n=0$ Mott phase. In this region the critical behavior should be governed by the linearized potential at $x_{c}$, cf. Eq. (64), and therefore by the corresponding RG scaling. Analogously to what observed at $\mu=0$, see Subs. IV C 5 , the transition region around $x_{c}$ is expected to enlarge as $\Delta x \sim l^{1 / 3}$ independently of $p$. This RG prediction is fully supported by the same numerical analysis outlined at the end of Subs. IV C 5, for several values of $p$.

\section{The von Neumann entanglement entropy}

Finally, we consider the von Neumann entanglement entropy, and, specifically, the half-lattice entanglement 


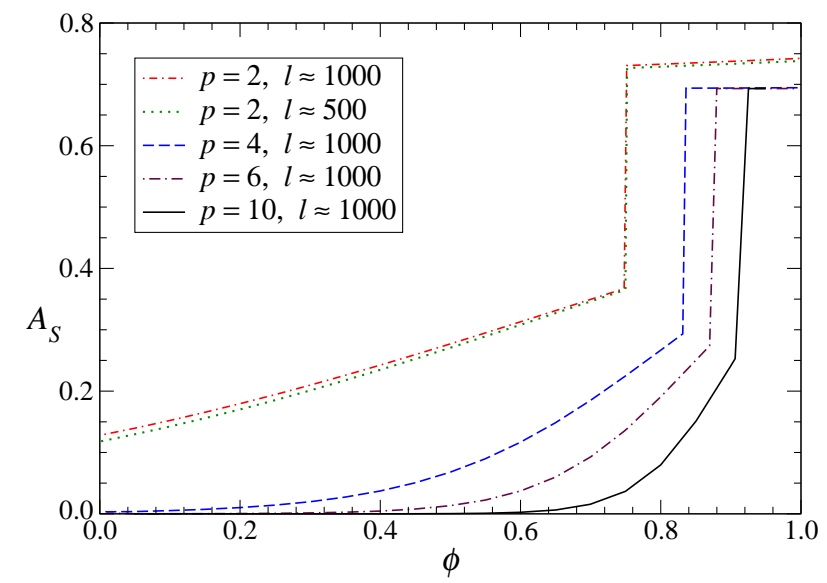

FIG. 30: The half-lattice von Neumann entanglement entropy $S_{1 / 2}$ at $\mu=-1$, for $p=2,4,6,10$. For $p=4, p=6$, and $p=10$, the plotted data are already very close to the large- $l$ limit.

entropy defined in Eq. (32) for chains with even $L$. We recall that the half-lattice entanglement entropy $S(L / 2 ; L)$ of the homogeneous $\mathrm{BH}$ model vanishes at $\mu=-1$, as it does at the low-density Mott transition point $\mu=1$, where it also vanishes in the presence of the trap, see Sec. II B 5. We instead find that at the $n=1$ Mott transition point $\mu=-1$ the presence of the trap gives rise to nonzero values depending of the phase-like variable $\phi$, i.e.,

$$
S_{1 / 2} \approx A_{S}(\phi)+O\left(l^{-\kappa}\right),
$$

with $\kappa$ roughly compatible with $\theta / 2$. Results for $p=$ $2,4,6,10$ are shown in Fig. 30. Notice that the discontinuity in $A_{s}$ occurs at $\phi=\phi_{0}=(p+1) /(p+2)$, cf. Eq. (85). The data suggest $A_{S}(\phi) \rightarrow 0$ as $p \rightarrow \infty$ (for $\phi<1$ ), consistently with the "naive" $p \rightarrow \infty$ limit of the homogeneous model with open boundary conditions.

\section{E. Universality of the modulated TSS}

We have shown that the $\mathrm{BH}$ model (1) is characterized by a modulated TSS at the $n=1$ Mott transition. A natural question concerns its universality, i.e., whether, beside the critical exponents, also the modulation is universal.

In order to investigate this issue, we again consider the XXZ model (33), and present results obtained by DMRG calculations for $j_{z}=-1 / 2, \mu=\mu_{c}=-1 / 2$ which corresponds to the $n=1$ Mott transitions, and various power laws of the confining potential. The values of the trap size we could reach, $l \lesssim 300$, are significantly smaller than those for the XX chain, which are $O\left(10^{3}\right)$.

The numerical results show periodic structures of the gap and the particle density analogous to those of the XX chain, see Fig. 17. We find again level crossings
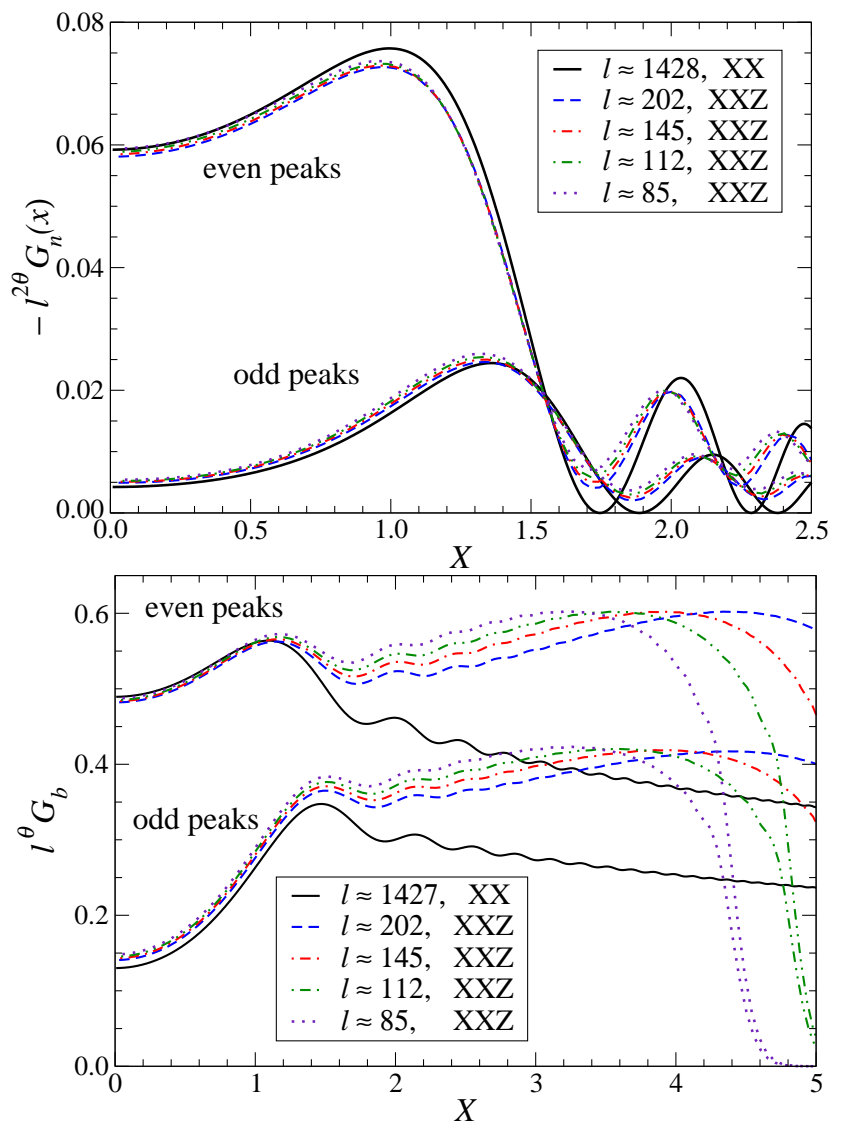

FIG. 31: The rescaled connected correlation $l^{2 \theta} G_{n}(x)$ (above) and the rescaled one-particle density matrix $l^{\theta} G_{b}(x)$ (below) vs. $X \equiv x l^{-\theta}$ for the XXZ model at $j_{z}=-1 / 2$ and $\mu=\mu_{c}$, $p=4$ and $l$ s corresponding to peaks of $\Delta$. For comparison, we also show results for the XX model for $\mu=-1, p=4$ and a large trap size, see also Sec. V B and C.

whose interval tends to a constant in the asymptotic large trap regime. The asymptotic interval between even level crossings, cf. Eq. (80), is given by $P_{l}^{*}=1.550$ for $p=2, P_{l}^{*}=1.293$ for $p=4$, and $P_{l}^{*}=1.200$ for $p=6$. Note that these values differ from those found for the XX model, see Eq. (81).

After introducing a variable $\phi$ defined as in Eq. (82), the gap turns out to behave analogously to the XX model, see Eq. (83). Results for the position $\phi_{0}$ of the odd zero are shown in Fig. 21: they are consistent with the asymptotic values found for the XX model, although they show a slower approach, which is likely $O\left(l^{-\theta}\right)$ (for the XX model it was $O\left(l^{-2 \theta}\right)$ for this particular quantity). Results for the large and small peaks of the gap, and their locations, are shown in Figs. 22 and 23, respectively for $p=2$ and $p=4$. They appear to converge toward the same asymptotic values of the XX model, with similar $O\left(l^{-\theta / 2}\right)$ corrections. The ratio $\Delta_{l} / \Delta_{s}$, which is independent of normalizations like $\phi_{0}$, appears to scale according to Eq. (86), and the large-l extrapolated values are consistent with those found for the XX chain, see Fig. 
24. The agreement for $p=4$ and $p=6$ is very good; for $p=2$, the data are clearly approaching the $\mathrm{XX}$ value, but the values of $l$ considered are not sufficiently large to provide a precise extrapolation.

The DMRG results for the particle density at the origin, are consistent with the scaling behavior (88) with a universal scaling function $\mathcal{D}_{0}(\phi)$, as also shown by the results at the peaks of the gap reported in Fig. 25.

The behaviors of the density-density correlations function and of the one-particle density matrix also support the universality of the modulated TSS. Examples are shown in Fig. 31 for $p=4$. With increasing $l$ the data appear to approach, although slowly, the same scaling curves found for the XX model. Note that the asymptotic curves of different models are expected to be universal apart from normalizations. This implies that they should match after an appropriate rescaling of the axes. Assuming universality, the results of Figs. 31 indicate that such rescalings are quite small for $p=4$.

In conclusion, the DMRG results for the XXZ model at $j_{z}=-1 / 2$ show a modulated TSS analogous to that observed for the XX model, thus supporting universality.

\section{CONCLUSIONS}

In this paper we have studied the quantum critical behaviors of confined particle systems described by the 1D $\mathrm{BH}$ model (1) in the presence of a power-law confining potential $V(r)=(r / l)^{p}$, at the transitions between the Mott and superfluid phases, and within the gapless superfluid phase. We have considered the hard-core limit, $U \rightarrow \infty$, of the 1D BH model, which allows us to study the effects of the confining potential by exact and very accurate numerical results. We have analyzed the various zero-temperature quantum critical behaviors within the TSS framework [23, 24]. In the following we list our main results.

(i) At the low-density Mott transition, the TSS limit can be analytically derived within the quadratic spinless fermion representation, leading to a Schrödinger-like equation for the lowest states. The RG scaling arguments leading to the TSS Ansatz (3) and (4), with critical exponents $z=2, \nu=1 / 2$, and $\theta=p /(p+2)$, are fully confirmed. We have computed the TSS of several observables extending the results presented in Ref. [23], and checked universality by DMRG calculations within the XXZ model (33), which corresponds to adding nearestneighbor density-density interactions in the $\mathrm{BH}$ model (1). The TSS functions show peculiar behaviors, whose main features, like the discontinuities in the scaling particle density (see, e.g., Fig. 1 for $p=2$ ), are clearly related to the quantum nature of the transition.

(ii) The trap-size dependence turns out to be more subtle in the region of parameters where the filling $f$ of the corresponding homogeneous system is nonzero. This is essentially due to the presence of an infinite number of level crossings of the lowest states when increas- ing the trap size. Nevertheless, the particle density of the $1 \mathrm{D}$ hard-core $\mathrm{BH}$ model approaches its LDA in the large- $l$ limit, i.e., the value of the particle density of the homogeneous system at the effective chemical potential $\mu_{\text {eff }}(x) \equiv \mu+(x / l)^{p}$. Corrections are suppressed by powers of the trap size, and show a nontrivial scaling behavior.

(iii) The level-crossing phenomenon gives rise to a new interesting scenario at the $n=1$ Mott transition, requiring a revision of the simplest TSS Ansatz (3) and (4) into a modulated TSS: the TSS is still controlled by the trap-size exponent $\theta=p /(p+2)$, as in the case of the low-density Mott transition, but it gets modulated by periodic functions of the trap size. Indeed, the gap turns out to behave as $\Delta \approx A_{\Delta}(\phi) l^{-2 \theta}\left[1+O\left(l^{-\theta / 2}\right)\right]$, where the amplitude $A_{\Delta}(\phi)$ is a periodic function of the trap size $l$, through the phase-like variable $\phi$ measuring the distance from the closest even level crossing, see Figs. 18, 19 and 20 for $p=2,4,6$ respectively. Modulated TSS is also observed for other observables, like the particle density and its correlators, and the one-particle density matrix. For example, the particle density $\left\langle n_{x}\right\rangle$ at a distance $x$ from the middle of the trap shows the asymptotic behavior $\left\langle n_{x}\right\rangle \approx \rho_{\text {lda }}(x / l)+l^{-\theta} \mathcal{D}(X, \phi)$ where $X=l^{-\theta} x$, and $\rho_{\text {lda }}(x / l)$ is the LDA of the particle density, cf. Eq. (37). DMRG computations for the XXZ model (33) at $j_{z}=-1 / 2$ and at the $n=1$ Mott transition show an analogous modulated TSS, supporting its universality. The modulated TSS shows another peculiar aspect of the quantum nature of the Mott transitions.

(iv) We have also studied the trap-size dependence in the gapless superfluid phase, whose corresponding continuum theory is a conformal field theory with $z=1$. In this region the asymptotic trap-size dependence turns out to be characterized by two length scales with different power-law divergence in the large trap-size limit. One of them scales as $\xi \sim l$ and describes the behavior of observables related to smooth modes, such as the halflattice entanglement; the other one scales as $\xi \sim l^{\zeta}$ with $\zeta=p /(p+1)$ and it is found in observables involving the modes at the Fermi scale $k_{F}=\pi f$, where $f$ is the filling of the homogeneous system. Moreover, the asymptotic power law behaviors appear modulated by periodic functions of the trap size. For example, the gap behaves as $\Delta \sim t(\phi) l^{-1}$ where $t(\phi)$ is the triangle function (49) and $\phi$ is a phase-like variable measuring the distance from the periodic level crossings, cf. Eq. (47). Some notable relations are found for the behavior of observables related to smooth modes, such us the gap, the density at the origin, and the half-lattice von Neumann entanglement entropy. Their asymptotic behavior in the presence of a confining potential can be derived from that of the homogeneous $\mathrm{BH}$ model with open boundary conditions which represents the $p \rightarrow \infty$ limit, by replacing the lattice size $L$ with the entanglement length scale defined from the von Neumann entanglement entropy.

The main features of the trap-size dependence reported in this paper should not be restricted to the hard-core 
limit, i.e., the limit $U \rightarrow \infty$ in the BH Hamiltonian (1). Specifically, the phenomenon of the level crossings at finite trap size should persists at finite values of $U$, because the total particle number is conserved by the confining potential even at finite $U$. A study of the trap-size dependence at the Mott transitions of the 1D BH model at finite $U$ would be important to further assess the extension of the universality of the modulated TSS observed in the hard-core limit. The presence of level crossings should also characterize the trap-size dependence in higher dimensions. Therefore, modulated TSS may be also found at higher dimensions.

Helpful discussions with P. Calabrese and M. Mintchev are gratefully acknowledged.

\section{Appendix A: Some details on numerical methods}

The numerical diagonalization of the Hamiltonian (8) is straightforward. Using lapack, $L=5000$ chains can be diagonalized in a few minutes on a desktop PC. We consider chains of size $L$ with open boundary conditions. The trap of size $l$ is centered in the middle of the chain. The size $L$ of the chain is taken sufficiently large to obtain results consistent with the infinite-size limit to machine precision.

As it is clear from the discussion of the previous sections, and specifically in Sec. III, the density $\left\langle n_{x}\right\rangle$ decreases very rapidly for $x>x_{\text {lda }} \equiv l(-1-\mu)^{1 / p}$ : it is sufficient to choose $L$ slightly larger than $2 x_{\text {Ida }}$ to have completely negligible finite-size effects. This allows us to obtain results for quite large $O\left(10^{3}\right)$ trap sizes. A few examples of values of $L$ giving boundary effects $\lesssim 10^{-15}$ for $p=2$ are: $\mu=0.999, l=10000: L=1200 ; \mu=0.9$, $l=1000: L=800 ; \mu=0, l=500: L=1100 ; \mu=-1$, $l=500: L=1600$. For larger values of $p$ boundary effects are smaller. Therefore, finite- $L$ effects at fixed trap size are under complete control. [34]

In the presence of the trap, most calculations are performed for odd $L \mathrm{~s}$ to have the trap centered in the middle site of the chain. We choose even $L$ s only to compute the trap-size dependence of the half-lattice von Neumann entanglement entropy. Notice that even and odd $L$ s may yield different results in the infinite-size limit, since the trap is centered between two chain sites and on a chain site respectively. For $\mu>-1$, this dependence on the parity on $L$ disappears very rapidly with increasing $l$ and it is totally negligible for $l \geq 20$ at $\mu=0$, for $l \geq 30$ at $\mu=-0.75$ and for $l \geq 120$ at $\mu=-0.9$. For $\mu=-1$, it must be taken into account, essentially because the role of the even and odd level crossings get interchanged, cf. Eqs. (82) and (84) in Sect. V A.

Numerical results for the XXZ model are obtained by finite-volume DMRG; the Hamiltonian is not translationinvariant, therefore the initialization of the procedure is slightly nonstandard. The number of states $M$ kept in the truncation procedure is chosen to have negligible truncation errors; for the largest chains we consid- ered $(L \cong 1000)$, we run with $M$ up to 140 , with discarded weights $<3 \times 10^{-9}$. Running at $L=999, p=2$, and $\mu=-1$ for a cycle of $\phi$, determining $l_{0}^{(554)}, l_{0}^{(555)}$, $l_{0}^{(556)}, l_{\text {peak }}^{(555)}$, and $l_{\text {peak }}^{(556)}$,cf. Subs. IV B, required about 100 runs for different $l, N$ combinations, each lasting about 8 hours.

\section{Appendix B: Finite-size behavior of the homogeneous 1D hard-core BH model with open boundary conditions}

In this section we report some exact results for the finite-size behavior of the homogeneous $1 \mathrm{D}$ hard-core $\mathrm{BH}$ model, or equivalently for the homogeneous XX chain, with open boundary conditions. This formally corresponds to the $p \rightarrow \infty$ limit of the confining potential, which becomes equivalent to a homogeneous chain of size $L=2 l$ with open boundary conditions. More precisely, the $p \rightarrow \infty$ limit of the $\mathrm{BH}$ model with the trap corresponds to a chain with an odd $L=2\lfloor l\rfloor+1(\lfloor x\rfloor$ is the largest integer not greater than $x$ ) when the center of the trap coincides with the middle site of the chain, and to an even $L=2\lfloor l\rfloor$ when the center is in the middle between two sites.

In the infinite-size limit $L \rightarrow \infty$, the filling $f$ is given by [25] $f \equiv\left\langle n_{i}\right\rangle=(1 / \pi) \arccos \mu$, thus in the range $1>\mu>-1$ we have $0<f<1$. Let us now consider a homogeneous system of finite size $L$ with open boundary conditions. The excitation number $N$ for $|\mu|<1$ is exactly given by

$$
N=\lfloor(L+1) f\rfloor,
$$

without any finite- $L$ correction. For integer $(L+1) f$, the ground state is degenerate $(\Delta=0)$; the lowest-energy simultaneous eigenvectors of the Hamiltonian and the particle number give $N=(L+1) f$ and $N=(L+1) f-1$.

For $f=1 / s$ with integer $s$, for every value of $N$ we have a vacuum degeneracy when $L+1=N s$, i.e., $\Delta=0$ for $L+1 \equiv 0(\bmod s)$. For $f=r / s$ with integer $r$ and $s$, again $\Delta=0$ for $L+1 \equiv 0(\bmod s)$, but we can satisfy $L+1=N s / r$ only for $N \equiv 0(\bmod r)$. For irrational $f$, $\Delta$ never vanishes for integer $L$.

Note that in the limit $\mu \rightarrow-1$, thus $f \rightarrow 1$, Eq. (B1) gives $N=L$ without vacuum degeneration; this is the expected result for $\mu \leq-1$. Analogously, Eq. (B1) gives $N=0$ for $\mu \rightarrow 1$ without vacuum degeneration, which is the expected result for $\mu \geq 1$.

Eq. (B1) suggests us to define

$$
\phi \equiv\{(L+1) f\},
$$

where $\{x\} \equiv x-\lfloor x\rfloor$ is the fractional part of $x$ (i.e., the sawtooth function). For integer $(L+1) f$, it is useful to label the two degenerate vacua with $\phi=0,1$ according to $N+\phi=(L+1) f$.

For each value of $\mu$, we observe that $L \Delta$ vs. $\phi$ collapses on a curve proportional to the triangle function $t(\phi)$ defined in Eq. (49), with $O(1 / L)$ corrections. Note that in 


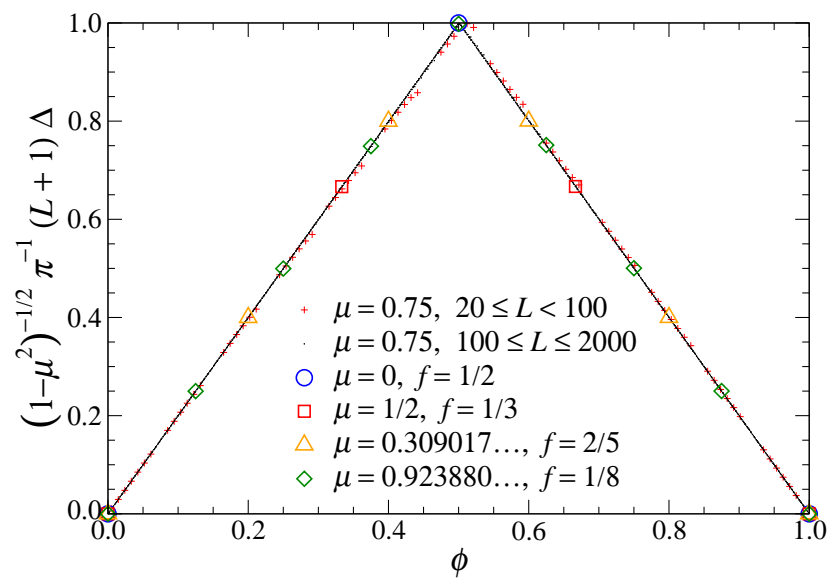

FIG. 32: The rescaled gap $\left(1-\mu^{2}\right)^{-1 / 2} \pi^{-1}(L+1) \Delta$ vs. $\phi$, defined in Eq. (B2), for the homogeneous system with open boundary conditions, for several values of $\mu$. The apparent solid line is due to the accumulation of data points for $\mu=$ $3 / 4$, corresponding to an irrational $f$. Data for rational $f$ are taken at $L \approx 1000$.

the case of rational filling $\phi$ takes only a discrete set of values; for $\mu=0$, e.g., $\phi$ takes the values 0 and $1 / 2$, corresponding to odd and even $L$ respectively. In Fig. 32 we plot data of $(L+1) \Delta$ for several values of $\mu$, corresponding to rational and irrational fillings (we use $(L+1) \Delta$ rather than $L \Delta$, which gives smaller, but comparable, finite- $L$ corrections). Thus we have the asymptotic behavior

$$
L \Delta=a_{\Delta} t(\phi)+O(1 / L)
$$

This corresponds to an asymptotic periodicity of the $L$ dependence of $L \Delta$ with period $1 / f$. The numerical results for $a_{\Delta}$ turn out to be perfectly reproduced by the simple formula

$$
a_{\Delta}=\pi\left(1-\mu^{2}\right)^{1 / 2}
$$

Note that $a_{\Delta}=0$ at $\mu= \pm 1$, indeed $\Delta=O\left(1 / L^{2}\right)$ at $\mu= \pm 1$ without level crossings, consistently with the fact that the corresponding continuum theory has $z=2$. For $|\mu|>1$ we instead have $\Delta=2(|\mu|-1)+O\left(L^{-2}\right)$.

We have also studied the particle density in the middle of the chain $\left\langle n_{0}\right\rangle$, which is only defined for odd $L$. We define

$$
\bar{\phi} \equiv 2\{[(L+1) f+1] / 2\}
$$

$0 \leq \bar{\phi} \leq 2$; note that either $\bar{\phi}=\phi$ or $\bar{\phi}=\phi+1$. Then we find that, at fixed $\mu$,

$$
(L+1)\left(\left\langle n_{0}\right\rangle-f\right)=1-\bar{\phi}
$$

without any finite- $L$ correction. We plot in Fig. 33 the r.h.s. of Eq. (B6) vs. $\bar{\phi}$, for several values of $\mu$; note that, for $f=r / s$ with integer $r$ and $s, \phi$ is limited to a discrete

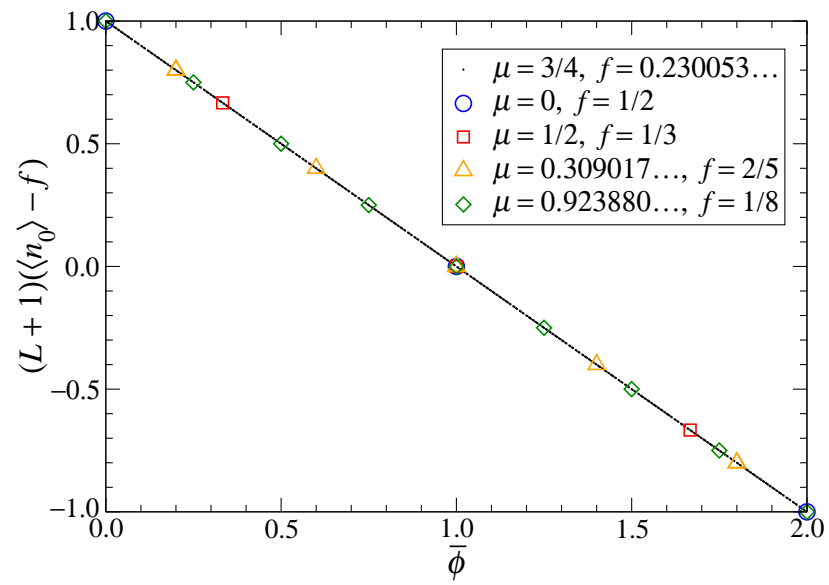

FIG. 33: The 1.h.s. of Eq. (B6) vs. $\bar{\phi}$, defined in Eq. (B5), for odd $L$ s in the range $3 \leq L \leq 999$.

set of $s$ values: $\{(2 i-1) / s, i=1, \ldots, s\}$ for odd $s$ and $\{(2 i-2) / s, i=1, \ldots, s\}$ for even $s$ (in this case, we also use $\bar{\phi}=2$, which is equivalent to $\bar{\phi}=0$, to distinguish the two vacua: $\bar{\phi}=2$ for the $N=(L+1) f$ vacuum and $\bar{\phi}=0$ for the $N=(L+1) f-1$ vacuum. On the other hand, for irrational $f$ s the values of $\phi$ are distributed all over the interval $(0,2)$.

For rational $f$, Eq. (B6) implies a very simple rational form for $\left\langle n_{0}\right\rangle$. For $\mu=0$, we have

$$
(L+1)\left(\left\langle n_{0}\right\rangle-\frac{1}{2}\right)= \begin{cases} \pm 1, & L \equiv 1(\bmod 4) \\ 0, & L \equiv 3(\bmod 4)\end{cases}
$$

where the \pm signs apply to the two degenerate lowest states with particle number $N=(L+1) / 2$ and $N=$ $(L-1) / 2$ respectively. For $\mu=1 / 2$, we have

$$
(L+1)\left(\left\langle n_{0}\right\rangle-\frac{1}{3}\right)= \begin{cases}\frac{2}{3}, & L \equiv 0(\bmod 3) \\ -\frac{2}{3}, & L \equiv 1(\bmod 3) \\ 0, & L \equiv 2(\bmod 3)\end{cases}
$$

Eqs. (B7) and (B8) are indeed verified for all $L$ s to machine precision. For other $\mu$ s giving rational $f$, we obtain comparable results.

For $\mu=0,\left\langle n_{i}\right\rangle$ is given by a very simple expression for all points $i=x+\frac{1}{2}(L+1), i=1, \ldots, L$ :

$$
(L+1)\left(\left\langle n_{i}\right\rangle-\frac{1}{2}\right)= \begin{cases}0, & \text { even } L, \\ 0, & \text { odd } L, \text { even } i, \\ \pm 1, & \text { odd } L, \text { odd } i\end{cases}
$$

where again the \pm signs apply to the two degenerate lowest analogously to Eq. (B7). Eq. (B9) for $i=\frac{1}{2}(L+1)$ gives back Eq. (B7).

Note that all the above formulae are invariant under the particle-hole exchange $n_{i} \rightarrow 1-n_{i}$, which implies $N \rightarrow L-N, f \rightarrow 1-f$, and $\mu \rightarrow-\mu$. 


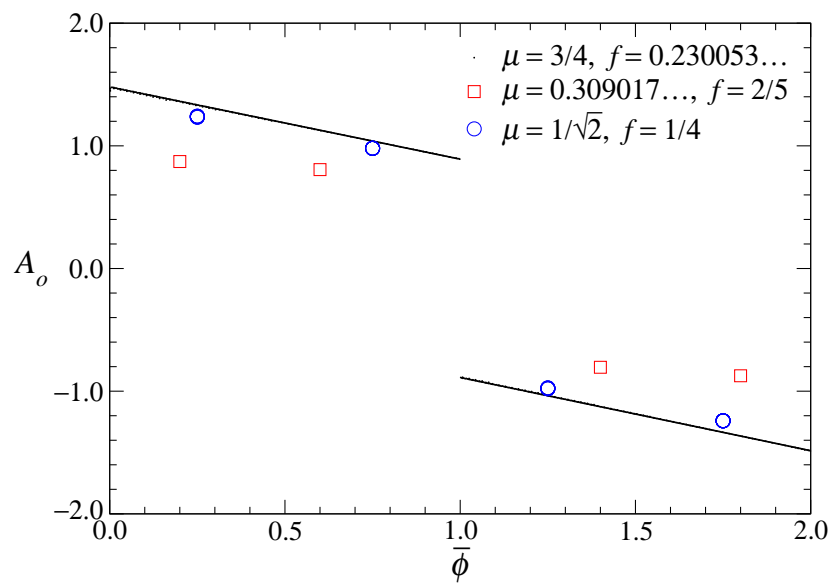

FIG. 34: The function $A_{o}(\mu, \bar{\phi})$ as derived from data of the quantity reported in Eq. (B14), vs. $\bar{\phi}$, cf. Eq. (B5), for several values of $\mu$. For $\mu=3 / 4$, all chains with even $200 \leq L \leq 2000$ are plotted. These results show that in all cases $A_{o}$ can be parametrized as in Eq. (B13).

We now consider the half-lattice von Neumann entanglement entropy $S(L / 2 ; L)$ for even $L$ and open boundary conditions in the superfluid phase, whose asymptotic large- $L$ behavior is [32]

$$
S(L / 2 ; L)=\frac{1}{6} \ln L+E(\mu)+O(1 / L) .
$$

The $O(1)$ term $E(\mu)$ depends on $\mu$; using the results reported in Refs. [28, 35], we derive

$$
\begin{aligned}
E(\mu) & =\frac{1}{12} \ln \left(1-\mu^{2}\right)+\frac{1}{6} \ln (4 / \pi) \\
& +\int_{0}^{\infty} d t\left(\frac{\cosh (t / 2)}{4 \sinh ^{3}(t / 2)}-\frac{e^{-t}}{6 t}-\frac{1}{2 t \sinh ^{2}(t / 2)}\right) .
\end{aligned}
$$

We recall that the von Neumann entanglement entropy $S(L / 2 ; L)$ vanishes at $\mu= \pm 1$.

An accurate numerical analysis using data up to $L=$ $O\left(10^{4}\right)$ shows that, for any $|\mu|<1$ including those corresponding to irrational filling, the entanglement entropy of the half-lattice behaves as

$$
S(L / 2 ; L)=\frac{1}{6} \ln (L+1)+E(\mu)+\frac{1}{L} A_{o}(\mu, \bar{\phi})+O\left(1 / L^{2}\right),
$$

where $\bar{\phi}$ is defined in Eq. (B5), and $A_{o}(\mu, \bar{\phi})$ has zero average over values of $\bar{\phi}$. $A_{o}$ can be written as

$$
A_{o}(\mu ; \bar{\phi})=\left\{\begin{array}{cc}
b+u \bar{\phi} & \text { for } \quad 0<\bar{\phi}<1 \\
-b-u(2-\bar{\phi}) & \text { for } \quad 1<\bar{\phi}<2
\end{array}\right.
$$

where $b$ and $u$ depend only on $\mu$. Indeed, in the case of irrational filling $f$, we find that, for sufficiently large $L$, the quantity

$$
L\left[S(L / 2 ; L)-\frac{1}{6} \ln (L+1)-E(\mu)\right]
$$

collapses on a single curve $A_{o}(\mu, \bar{\phi})$ at fixed $\mu$, given by Eq. (B13), with $O(1 / L)$ corrections. In the case of rational $f$, we have a discrete set of possible values of $\bar{\phi}$, and the data accumulate at points located along two lines as described by Eq. (B13). Some results are shown in Fig. 34. We find $b \cong 1.48, u \cong-0.59$ for $\mu=3 / 4$; $b \cong 1.37, u \cong-0.52$ for $\mu=1 / \sqrt{2} ; b \cong 0.91, u \cong-0.17$ for $\mu=\cos (2 \pi / 5) \cong 0.309017$.

We mention that subleading oscillations in the behavior of entanglement entropies have been also reported in other studies, see, e.g., Refs. [36, 37].

In the case $\mu=0$, the values of $\bar{\phi}$ corresponding to even $L$ are restricted to $\bar{\phi}=1 / 2,3 / 2$, which implies $A_{o} \sim(-1)^{L / 2}$. More precisely, our numerical results are accurately reproduced by the formula

$$
\begin{aligned}
S(L / 2 ; L)= & \frac{1}{6} \ln (L+1)+E(0)-(-1)^{L / 2} \frac{\pi}{4(L+1)} \\
& +O\left(1 / L^{2}\right),
\end{aligned}
$$

with $E(0)=0.28776969994598 \ldots$, cf. Eq. (B11). This expression for the half-lattice entanglement entropy is consistent with an analogous formula reported in Ref. [36] for the XX model at $\mu=0$. The $O\left(1 / L^{2}\right)$ term remaining in Eq. (B15) is very small $\left(\sim 10^{-5} L^{-2}\right)$ and without oscillations within numerical precision $\left[\sim 10^{-15}\right.$ on $\left.S(L / 2 ; L)\right]$.
[1] I. Bloch, J. Dalibard, and W. Zwerger, Rev. Mod. Phys. 80, 885 (2008).

[2] M. Greiner, I. Bloch, M.O. Mandell, T. Hänsch, and T. Esslinger, Nature 415, 39 (2002).

[3] T. Stöferle, H. Moritz, C. Schori, M. Köhl, and T. Esslinger, Phys. Rev. Lett. 92, 130403 (2004).

[4] B. Paredes, A. Widera, V. Murg, O. Mandel, S. Fölling, I, Cirac, G. Shlyapnikov, R.W. Hänsch, and I. Bloch, Nature 429, 277 (2004).

[5] T. Kinoshita, T. Wenger, and D.S. Weiss, Science 305, 1125 (2004); Phys. Rev. Lett. 95, 190406 (2005).

[6] Z. Hadzibabic, P. Krüger, M. Cheneau, B. Battelier, and
J. Dalibard, Nature 441, 1118 (2006).

[7] S. Fölling, A. Widera, T. Müller, F. Gerbier, and I. Bloch, Phys. Rev. Lett. 97, 060403 (2006).

[8] I.B. Spielman, W.D. Phillips, and J.V. Porto, Phys. Rev. Lett. 98, 080404 (2007); Phys. Rev. Lett. 100, 120402 (2008).

[9] D. Clément, N. Fabbri, L. Fallani, C. Fort, and M. Inguscio, Phys. Rev. Lett. 102, 155301 (2009).

[10] D. Jaksch, C. Bruder, J.I. Cirac, C.W. Gardiner, and P. Zoller, Phys. Rev. Lett. 81, 3108 (1998).

[11] M.P.A. Fisher, P.B. Weichmann, G. Grinstein, and D.S. Fisher, Phys. Rev. B 40, 546 (1989). 
[12] A. Pelissetto and E. Vicari, Phys. Rep. 368, 549 (2002).

[13] G.G. Batrouni, V. Rousseau, R.T. Scalettar, M. Rigol, A. Muramatsu, P.J.H. Denteneer, and M. Troyer, Phys. Rev. Lett. 89, 117203 (2002).

[14] V.A. Kashurnikov, N.V. Prokofev, and B.V. Svistunov, Phys. Rev. A 66, 031601 (2002).

[15] C. Kollath, U. Schollwöck, J. von Deft, W. Zwerger, Phys. Rev. A 69, 031601 (2004).

[16] L. Pollet, S. Rombouts, K. Heyde, and J. Dukelsky, Phys. Rev. A 69, 043601 (2004).

[17] S. Wessel, F. Alet, M. Troyer, and G.G. Batrouni, Phys. Rev. A 70, 053615 (2004).

[18] M. Rigol and A. Muramatsu, Phys. Rev. A 70, 031603 (2004); Phys. Rev. A 72, 013604 (2005).

[19] B. DeMarco, C. Lannert, S. Vishveshwara, and T.-C. Wei, Phys. Rev. A 71, 063601 (2005).

[20] O. Gygi, H.G. Katzgraber, M. Troyer, S. Wessel, and G.G. Batrouni, Phys. Rev. A 73, 063606 (2006).

[21] L. Urba, E. Lundh, and A. Rosengren, J. Phys. B 39, 5187 (2006).

[22] M. Rigol, G.G. Batrouni, V.G. Rousseau and R.T. Scalettar, Phys. Rev. A 79, 053605 (2009).

[23] M. Campostrini and E. Vicari, Phys. Rev. A 81, 023606 (2010).

[24] M. Campostrini and E. Vicari, Phys. Rev. Lett. 102, 240601 (2009).

[25] S. Sachdev, Quantum Phase Transitions (Cambridge
Univ. Press, 1999).

[26] A.M. Tsvelik, Quantum Field Theory in Condensed Matter Physics, (Cambridge Univ. Press, 1995).

[27] A.P. Young and H. Rieger, Phys. Rev. B 53, 8486 (1996).

[28] B.-Q. Jin and V.E. Korepin, J. Stat. Phys. 116, 79 (2004).

[29] I. Peschel, J. Phys. A 36, L205 (2003).

[30] S. Bergkvist, P. Henelius, and A. Rosengren, Phys. Rev. A 70, 053601 (2004).

[31] S.M. Pittman, G.G. Batrouni, and R.T. Scalettar, Phys. Rev. B 78, 214208 (2008).

[32] P. Calabrese and J. Cardy, J. Stat. Mech. P06002 (2004); J. Phys. A 42, 504005 (2009).

[33] V. Eisler, F. Igói, and I. Peschel, J. Stat. Mech. P02011 (2009).

[34] Finite- $L$ effects in the presence of the trap, and, specifically, in the trap-size scaling limit, have been considered in S.L.A. de Queiroz, R.R. dos Santos, and R.B. Stinchcombe, arXiv:1003.1075, in the context of classical spin models.

[35] H.-Q. Zhou, T. Barthel, J. Fiaerestad, and U. Schollwöck, Phys. Rev. A 74, 050305 (2006).

[36] N. Laflorencie, E.S. Sorensen, M.-S. Chang, and I. Affleck, Phys. Rev. Lett. 96, 100603 (2006).

[37] P. Calabrese, M. Campostrini, F. Essler, and B. Nienhuis, Phys. Rev. Lett. in press, arXiv:0911.4660. 